\title{
Role of glucosyltransferase $R$ in biofilm interactions between Streptococcus oralis and Candida albicans
}

\author{
João Gabriel Silva Souza ${ }^{1,2}$ - Martinna Bertolini ${ }^{1} \cdot$ Angela Thompson $^{1} \cdot$ Jillian M. Mansfield $^{3}$. \\ André Alex Grassmann ${ }^{4} \cdot$ Kendra Maas ${ }^{5}$. Melissa J. Caimano ${ }^{4}$. Valentim Adelino Ricardo Barao ${ }^{2}$. \\ M. Margaret Vickerman ${ }^{3} \cdot$ Anna Dongari-Bagtzoglou'
}

Received: 21 October 2019 / Revised: 8 January 2020 / Accepted: 30 January 2020 / Published online: 10 February 2020

(c) The Author(s) 2020. This article is published with open access

\begin{abstract}
Streptococcal glucosyltransferases (Gtf) synthesize $\alpha$-glucan exopolymers which contribute to biofilm matrix. Streptococcus oralis interacts with the opportunistic pathogen Candida albicans to form hypervirulent biofilms. S. oralis 34 has a single gtf gene $(g t f R)$. However, the role of $g t f R$ in single and mixed species biofilms with $C$. albicans has never been examined. A $g t f R$ deletion mutant, purified GtfR, and recombinant GtfR glucan-binding domain were tested in single and mixed biofilms on different substrata in vitro. A mouse oral infection model was also used. We found that in single species biofilms growing with sucrose on abiotic surfaces $S$. oralis $g t f R$ increased biofilm matrix, but not bacterial biomass. In biofilms with C. albicans, $S$. oralis encoding $g t f R$ showed increased bacterial biomass on all surfaces. C. albicans had a positive effect on $\alpha$-glucan synthesis, and $\alpha$-glucans increased $C$. albicans accretion on abiotic surfaces. In single and mixed infection of mice receiving sucrose $S$. oralis gtfR enhanced mucosal burdens. However, sucrose had a negative impact on $C$. albicans burdens and reduced $S$. oralis burdens in co-infected mice. Our data provide new insights on the GtfR-mediated interactions between the two organisms and the influence of biofilm substratum and the mucosal environment on these interactions.
\end{abstract}

Supplementary information The online version of this article (https:// doi.org/10.1038/s41396-020-0608-4) contains supplementary material, which is available to authorized users.

M. Margaret Vickerman mmv4@buffalo.edu

$\triangle$ Anna Dongari-Bagtzoglou adongari@uchc.edu

1 Department of Oral Health and Diagnostic Sciences, University of Connecticut School of Dental Medicine, Farmington, CT, USA

2 Department of Prosthodontics and Periodontology, Piracicaba Dental School, University of Campinas (UNICAMP), Piracicaba, São Paulo, Brazil

3 Department of Oral Biology, School of Dental Medicine, University at Buffalo, Buffalo, NY, USA

4 Departments of Medicine, Pediatrics and Molecular Biology and Biophysics, University of Connecticut Health Center, Farmington, CT, USA

5 Microbial Analysis, Resources, and Services Core, University of Connecticut, Storrs, CT, USA

\section{Introduction}

Glucosyltransferases (Gtfs) are streptococcal extracellular or cell-associated exoenzymes that hydrolyze sucrose and synthesize glucan polymers from the resulting glucose $[1,2]$. These extracellular polymers, produced in various proportions of $\alpha-1,6$ and $\alpha-1,3$-linkages [3], contribute to the matrix that forms the scaffold for the three-dimensional architecture of biofilms, increasing bacterial adhesion, coaggregation, and tolerance against antimicrobials [4-8]. Growth in the biofilm state occurs in many bacterial infectious diseases, thus glucans synthesized by Gtfs have been implicated as virulence factors [8].

Several oral streptococcal species have Gtf enzymes that may affect biofilm growth on abiotic, tooth, and mucosal surfaces. Although the Gtfs of Streptococcus mutans have been well characterized, due to their role in dental caries development, the biological roles of Gtf from the mitis group streptococci, including $S$. oralis, in oral biofilm communities are less clear. Streptococci of this group have been recognized as main initial colonizers in biofilms formed on tooth surfaces [9-11] and glucans have been implicated in facilitating biofilm accumulation [12, 13]. 
Members of the mitis group have a single Gtf-encoding gene, often regulated by a co-transcribed positive regulator, $\operatorname{Rgg}[13,14]$. Mitis group streptococci have been termed "accessory pathogens" due to their ability to form multispecies biofilms and enhance the community virulence [15].

Candida albicans-streptococcal interactions play an important role during the course of oral opportunistic infections [16-19]. Co-infection with $S$. oralis and C. albicans in a mouse model increased bacterial biofilms and severity of mucosal lesions, enhanced fungal pathogenicity, and resulted in an exaggerated inflammatory response [18]. S. oralis has a single Gtf structural gene ( $g t f R$ ) [13]. GtfR has a high homology to other Gtf enzymes from mitis group streptococci, being able to synthesize water soluble and insoluble glucans using sucrose as substrate [13]. However, the role of GtfR and its $\alpha$-glucan products in biofilm phenotypes has not been tested experimentally. We hypothesized that $\alpha$-glucans synthesized by GtfR increase $S$. oralis biomass in biofilms growing with sucrose. We also hypothesized that GtfR-derived $\alpha$-glucans modulate cross-kingdom interactions between $S$. oralis and C. albicans that lead to mutualistic relationships in mixed biofilms. In addition, because the type of substratum can affect microorganism adhesion and biofilm phenotype [20], we tested whether GtfR-mediated cross-kingdom interactions could be modulated by the type of biofilm substratum or by the mucosal environment in vivo.

\section{Materials and methods}

\section{Strains and growth conditions}

Strain construction in the $S$. oralis 34 parental background was done using standard molecular cloning techniques with modifications for oral streptococcal species [21] as described in Supplemental Material. S. oralis strains were reactivated from glycerol stocks by overnight growth in brain-heart infusion (BHI) medium (Becton, Dickinson and Company, Sparks, MD, USA) supplemented with antibiotics (Spectinomycin, $250 \mu \mathrm{g} / \mathrm{ml}$, Erythromycin, $5 \mu \mathrm{g} / \mathrm{ml}$ ) as needed, under static conditions at $37{ }^{\circ} \mathrm{C}$, in a $5 \% \mathrm{CO}_{2}$ incubator. C. albicans strain SC5314 was used as it forms robust biofilms with $S$. oralis 34 on abiotic and mucosal substrata and was grown as detailed previously [19].

\section{Single and mixed biofilm growth on abiotic surfaces}

Biofilms of streptococci and C. albicans were allowed to develop for $6-48 \mathrm{~h}$ in RPMI 1640 medium supplemented with $10 \%$ FBS and $10 \%$ BHI $[22,23]$. In $48 \mathrm{~h}$ biofilms, fresh media were added after $24 \mathrm{~h}$. In some experiments media were supplemented with $1-5 \%$ [wt/vol] sucrose or
$1 \%$ glucose [24]. Biofilms were grown on polystyrene (12well plates and chamber slides) or titanium disks placed within the wells of 12-well tissue culture plates (American Society for Testing and Materials grade 2). Where indicated, surfaces were coated with FBS or dextran $(100 \mu \mathrm{g} /$ $\mathrm{ml}$ ), for $30-60 \mathrm{~min}$ at $37^{\circ} \mathrm{C}$. Adhesion to coated and uncoated abiotic surfaces was assessed $1 \mathrm{~h}$ post inoculation, after washing nonadherent cells.

For biofilm growth, overnight stationary-phase cultures of S. oralis were inoculated in fresh BHI broth, allowed to reach exponential growth, and adjusted to $\mathrm{OD}_{600}=1$, representing a final suspension of $10^{7} \mathrm{cells} / \mathrm{ml}$. Overnight cultures of $C$. albicans were prepared in YPD broth. The final inoculum in each biofilm consisted of $10^{5}$ cells of C. albicans and $10^{7}$ cells of $S$. oralis. In some experiments WT and $\Delta g t f R$ strain biofilms were allowed to form for $24 \mathrm{~h}$. Subsequently biofilms were washed with PBS and fresh media containing an inoculum of $C$. albicans $\left(10^{5}\right.$ yeast cells) were added and incubated for up to $16 \mathrm{~h}$. Biofilms were incubated under static conditions at $37^{\circ} \mathrm{C}$ in a $5 \% \mathrm{CO}_{2}$ incubator.

\section{Biofilm growth on oral mucosal surfaces in vitro and in vivo}

To examine the role of $\alpha$-glucans in mucosal biofilm growth an oral stratified nonkeratinizing mucosal analog was used, described in detail previously $[25,26]$. Tissues were infected with $C$. albicans $\left(10^{6}\right.$ cells/tissue), S. oralis 34 strains (WT and $\Delta g t f R$ mutant, $10^{7}$ cells/tissue) or a combination, in media supplemented with $1 \%$ sucrose [19]. Single and mixed biofilm growth was tested in vivo using a previously published mouse oral infection model, as described in Supplementary Methods [18].

\section{Microscopic analyses of biofilms}

In some experiments Alexa Fluor 647-labeled dextran conjugate $(1 \mu \mathrm{M}$; absorbance/fluorescence emission maxima, $647 / 668 \mathrm{~nm}$ ) was added during biofilm growth, which is incorporated into $\alpha$-glucans during matrix formation [27]. Biofilms were fixed with $4 \%$ paraformaldehyde for $2 \mathrm{~h}$ at $4{ }^{\circ} \mathrm{C}$. C. albicans was visualized after staining for $2 \mathrm{~h}$ at room temperature using an FITC-labeled anti-Candida polyclonal antibody (Meridian Life Science, ME, USA). For biofilms containing streptococci, this was followed by fluorescence in situ hybridization with the EUB338 probe labeled with Alexa 405 or with Alexa 633-labeled probe [28, 29]. Biofilms were visualized by confocal microscopy. Stacks of $z$-plane images from at least three different fields of view per sample were reconstructed into 3-D images using IMARIS (Bitplane, Inc., Saint Paul, MN, USA). Surface reconstructions were used to calculate biovolumes and thickness. To visualize biofilms on mucosal constructs, tissue sections were stained 
as described above, and counterstained with Hoechst 33258 (Invitrogen, Carlsbad, CA, USA) to visualize epithelial nuclei. Biofilms growing on the tongue surface of infected mice were examined by scanning electron microscopy as described in Supplementary Material.

\section{Fungal and bacterial viable counts}

Biofilms were vigorously vortexed in $2 \mathrm{ml}$ PBS for $10 \mathrm{~s}$, followed by sonication at $7 \mathrm{~W}$ for $30 \mathrm{~s}$ to break up cell aggregates. Mucosal tissues were homogenized followed by gentle sonication. Sonicates were serially diluted in PBS and $40 \mu \mathrm{l}$ aliquots were plated on BHI agar supplemented with Nystatin $(250 \mathrm{U} / \mathrm{ml})$ for $S$. oralis $34 \mathrm{WT}$ and on Sabouraud Dextrose Agar supplemented with chloramphenicol $(1 \mathrm{mg} / \mathrm{ml})$ for C. albicans quantification. For $\Delta g t f R$ strain plates were additionally supplemented with spectinomycin. S. oralis plates were incubated at $37^{\circ} \mathrm{C}$ in an atmosphere of $5 \% \mathrm{CO}_{2}$ and Candida plates at $30^{\circ} \mathrm{C}$ in aerobic conditions for 2 days. Colony-forming units (CFUs) were counted by stereomicroscopy, and the results were expressed as log CFUs per biofilm.

\section{Gene expression analyses}

Streptococcal RNA was purified from biofilms according to a published protocol which reduces the amount of extracellular polymers in biofilm matrix that interfere in RNA extraction [30]. The gyrase gene (gyrA) was used as internal control, which is stably expressed gene in $C$. albicans-streptococcal biofilms [31]. Data were calculated by the $\Delta \Delta \mathrm{Cq}$ method and $g t f R$ gene expression in co-species biofilms was expressed as fold relative to single species $S$. oralis biofilms [31].

C. albicans RNA from biofilms was extracted according to our previous protocol [22, 23]. For mucosal biofilms, tissues were homogenized using a POLYTRON ${ }^{\circledR}$ homogenizer and homogenates were beat with $0.5 \mathrm{~mm}$ zirconium beads (BeadBug ${ }^{\circledR}$ prefilled tubes, Sigma-Aldrich, St. Louis, MO, USA). Efbl gene was used as housekeeping control. Hyphae-associated genes als $3, h w p l$, and efgl were analyzed using primer sequences previously described [23].

\section{GtfR purification}

GtfR secreted by wild-type $S$. oralis 34 was purified by chromatography according to a protocol [13] that results in active enzyme, as detailed in Supplementary Methods. Glucan synthesis was tested by coating polystyrene surfaces with purified enzyme ( $1 \mu \mathrm{g} / \mathrm{ml}$ in carbonate-bicarbonate buffer) for $1 \mathrm{~h}$ at $37^{\circ} \mathrm{C}$, followed by overnight incubation with Alexa Fluor 647-labeled dextran conjugate $(1 \mu \mathrm{M})$, supplemented with $1 \%$ sucrose. A solution containing the fluorescently labeled probe but no sucrose served as negative control.

\section{Cloning and heterologous expression of recombinant GtfR glucan-binding domain}

To assess the role of the glucan-binding domain (GBD) of GtfR in biofilms we generated a recombinant GBD (rGBD) in an Escherichia coli heterologous expression system. The 3-prime end of gtfR (encoding amino acids 1083 to 1554 of the 1575-residue GtfR protein) was amplified by PCR with primers NdeSo34GBDF and BamEngstopSo34GBDR2 (Table S1) and cloned in-frame with the compatibly digested E. coli expression vector pET28a for expression of rGBD with an $\mathrm{N}$-terminal $6 \times$ His tag. Expression and purification of rGBD are described in Supplementary Methods.

\section{Evaluation of $C$. albicans interactions with purified native GtfR and rGBD}

To test the ability of native GtfR to modify Candida adhesion in a sucrose-independent manner, polystyrene wells were coated with purified protein as above and C. albicans $\left(10^{5}\right.$ yeast cells/well, suspended in RPMI, 10\% FBS, 10\% BHI) was added for $1 \mathrm{~h}$. Surface area of adherent cells, stained with an FITC-labeled polyclonal antibody, was quantified microscopically using Image J. To test the sucrose-dependent GtfR effect on Candida adhesion $1 \%$ sucrose was added after coating, and glucans were allowed to form for $1 \mathrm{~h}$ at $37^{\circ} \mathrm{C}$. Wells were subsequently washed to remove residual sucrose, C. albicans was added and adhesion was assessed as above. Noncoated surfaces were used as control.

To test the ability of purified GtfR to adsorb to a preformed $C$. albicans biofilm and synthesize $\alpha$-glucans, biofilms grown for $24 \mathrm{~h}$ on polystyrene wells were incubated for $1 \mathrm{~h}$ with $10 \mu \mathrm{g} / \mathrm{ml}$ of purified protein in carbonatebicarbonate buffer [32]. After washing the unbound protein, glucans synthesized in situ were labeled by adding a solution containing Alexa Fluor 647-labeled dextran conjugate $(1 \mu \mathrm{M})$ supplemented with $1 \%$ sucrose, incubated overnight, and examined by confocal microscopy.

To test the binding activity of $r G B D$ to $C$. albicans, overnight YPD broth cultures $\left(10^{7}\right.$ yeast cells) were centrifuged and resuspended in PBS containing increasing concentrations of rGBD for $1 \mathrm{~h}$ at $37^{\circ} \mathrm{C}$. Cells were stained with 1:500 dilution of anti- $6 \times$ His-tag antibody conjugated to FITC (Biomatik ${ }^{\circledR}$, Wilmington, DE, USA) and visualized by fluorescence microscopy and flow cytometry. rGBD binding to $24 \mathrm{~h}$ preformed $C$. albicans biofilms was tested similarly.

\section{S rRNA gene high-throughput sequencing}

Tongue bacterial DNA and sequencing was performed using a lysis protocol and sequencing analysis pipeline optimized for mucosal microbiome characterization in murine tongue tissues [33]. The V4 region was amplified 
using $515 \mathrm{~F}$ and $806 \mathrm{R}$ primers with Illumina adapters and bar codes on the $3^{\prime}$ end. Sequencing and data analysis details are listed in Supplementary Methods. All 16S V4 DNA sequencing raw data have been deposited in NCBI, SRA accession: PRJNA593873. Data are accessible at the following link: http://www.ncbi.nlm.nih.gov/bioproject/ 593873.

\section{Statistics}

The Graph-Pad Prism software (Graphpad, La Jolla, CA, USA) was used for statistical analyses. Pair-wise and multiple group comparisons were done with the Bonferroni $t$-test and ANOVA, respectively, with significance set at $p<0.05$.

\section{Results}

\section{S. oralis glucosyltransferase affects biofilm structure on polystyrene surfaces}

To test whether $g t f R$ affects $S$. oralis biofilm formation and structure we first evaluated biofilm growth of $g t f R$ mutant and complemented strains on polystyrene surfaces. To rule out intrinsic adhesion differences among these strains that could contribute to an altered biofilm phenotype, we allowed strains to adhere to either FBS-coated, dextrancoated, or uncoated polystyrene surfaces for $1 \mathrm{~h}$, and then quantified attached organisms by CFU counts. We found no differences in adhesion between wild-type and mutant strains, although the complemented strain adhered more avidly to all surfaces (Fig. S1A).

To assess $\alpha$-glucan synthesis biofilm media were supplemented with sucrose as GtfR substrate, or glucose as negative control. Biofilms of parental and complemented strains growing in sucrose showed bacterial clusters enmeshed by $\alpha$-glucan matrix in $24 \mathrm{~h}$ and $48 \mathrm{~h}$ biofilms (Fig. 1a). Under the same growth conditions $\Delta g t f R$ mutants were able to form biofilms, albeit devoid of $\alpha$-glucans (Fig. 1a). Because the extracellular matrix is an important component of streptococcal biofilms [8], glucan production contributed to an increase in the total biofilm biovolume in both the wild type and complemented, as compared with the $\Delta g t f R$ strains (Fig. 1b). Parental and complemented strains formed biofilms with greater thickness (Fig. 1c, $24 \mathrm{~h}$ ) and density, due to the presence of the extracellular matrix filling spaces between cell clusters (Fig. 1a). Differences in total biofilm biovolume in parental, gtfR deletion and complemented strains growing with sucrose could be explained solely by the observed differences in their glucan matrix biovolumes, because viable bacterial counts were not significantly different in these biofilms (Fig. 1d). A higher total biofilm
(Fig. 1b) and matrix biovolume (Fig. 1e) were noted in the complemented compared with the wild-type strain, likely because it has multiple copies of plasmid-encoded $g t f R$ and expresses higher levels of this gene (Fig. S1B).

\section{In biofilms with C. albicans, S. oralis gtfR promotes bacterial matrix and biomass}

Growth of wild-type and complemented strains with C. albicans on polystyrene in $1 \%$ sucrose led to the development of dense biofilms enmeshed in $\alpha$-glucan-rich matrix (Fig. 2a). Confocal images of mixed biofilms suggested physical proximity between $\alpha$-glucan and $C$. albicans cells in $48 \mathrm{~h}$ biofilms (Fig. 2a). In contrast, mixed biofilms with the $\Delta g t f R$ mutant were sparsely distributed, although their thickness was similar to the wild-type strain (Fig. S1C). As expected, $\alpha$-glucan synthesis by the WT and complemented strains contributed to a higher total mixed biofilm biovolume compared with the $\Delta g t f R$ strain (Fig. 2b).

In $24 \mathrm{~h}$ biofilms growing in $1 \%$ sucrose we found a significant, greater than twofold increase in $S$. oralis counts with all three strains in mixed biofilms compared with single $S$. oralis biofilms, suggesting that the positive effect of $C$. albicans on $S$. oralis biomass does not require GtfR activity. However, this effect was significantly higher in the wild-type and complemented strains compared with the $\Delta g t f R$ mutant, showing that even though $g t f R$ is not required, it significantly enhanced this effect (Fig. 2c). In $48 \mathrm{~h}$ biofilms there was no significant effect of $C$. albicans on $S$. oralis viable counts for any strain, suggesting that differences in total biovolumes of late mixed biofilms among the three strains (Fig. 2b) are primarily due to differences in the amount of matrix produced. To further elucidate whether $C$. albicans has an effect on matrix production we compared single and mixed biofilms with the wild-type strain. The matrix biovolume in mixed biofilms with $C$. albicans was significantly higher compared with single $S$. oralis biofilms at $48 \mathrm{~h}$, and although the average biovolume was higher at $48 \mathrm{~h}$ compared with $24 \mathrm{~h}$ this difference was not significant (Fig. 2d). The matrix increase in mixed over single biofilms at $48 \mathrm{~h}$ was not due to higher $g t f R$ gene expression levels because expression was not different in mixed compared with single biofilms (Fig. 2e). These data suggest that on polystyrene surfaces $C$. albicans promotes an increase in streptococcal cell numbers in early stages of biofilm growth, leading to increased $\alpha$-glucan-rich matrix synthesis in late stages. This work also shows the positive influence of $C$. albicans on $S$. oralis growth on polystyrene is primarily $g t f R$-dependent.

We next asked whether $g t f R$ affects $C$. albicans growth in mixed biofilms. $S$. oralis strains conferred a modest growth advantage to $C$. albicans in $24 \mathrm{~h}$ biofilms, although this effect was more pronounced in biofilms with the 
A)

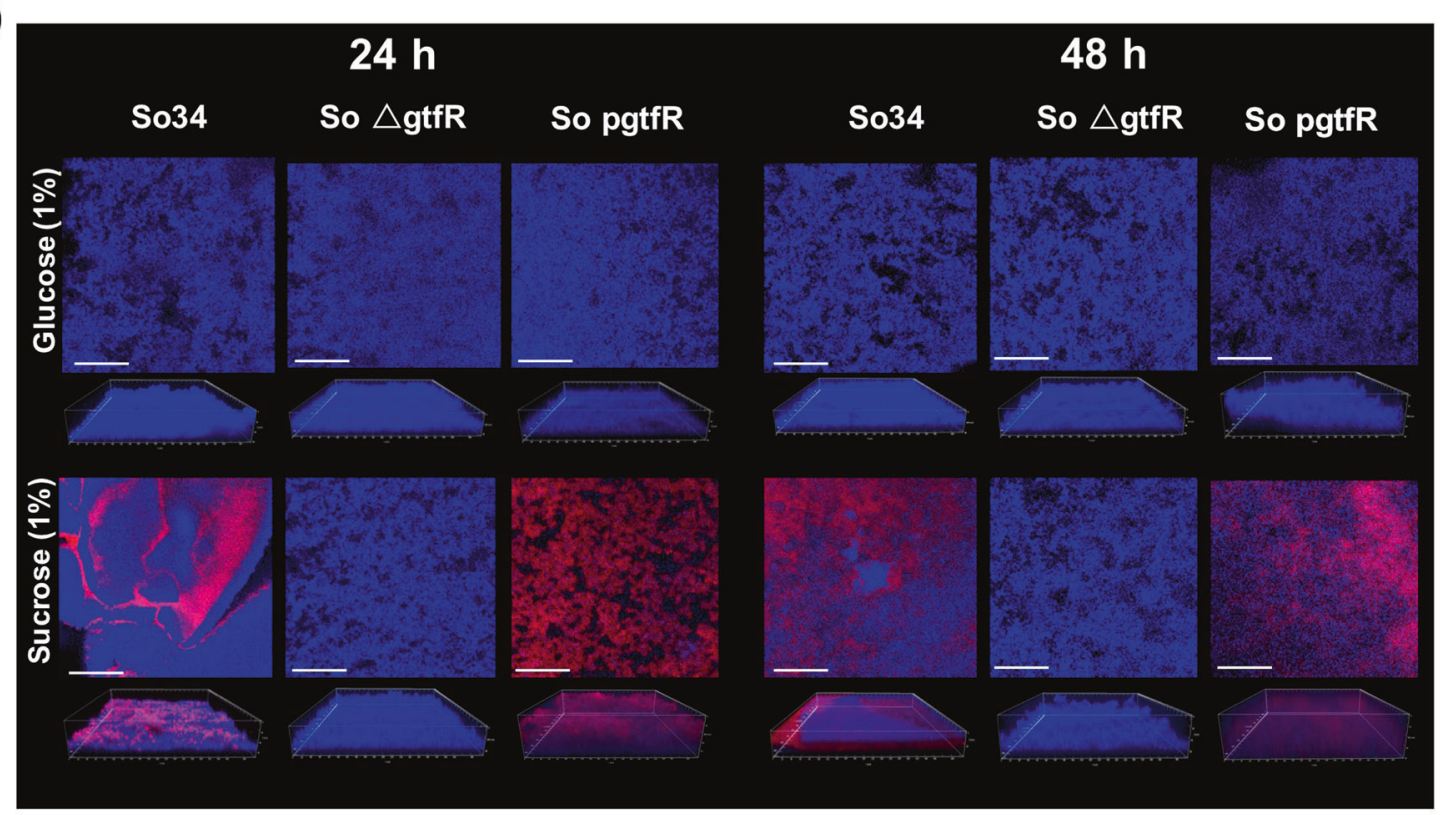

B)

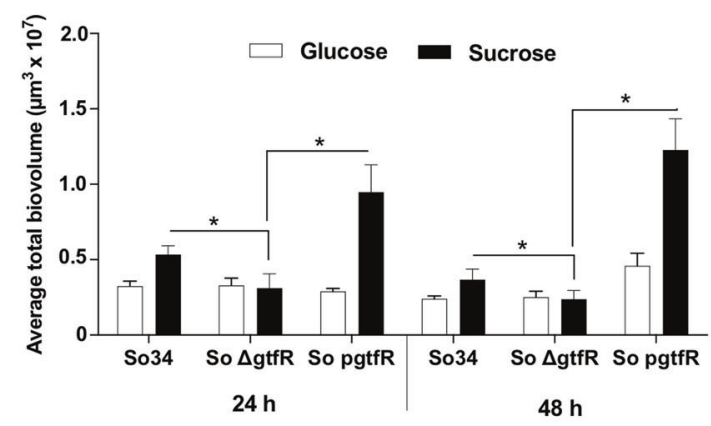

D)

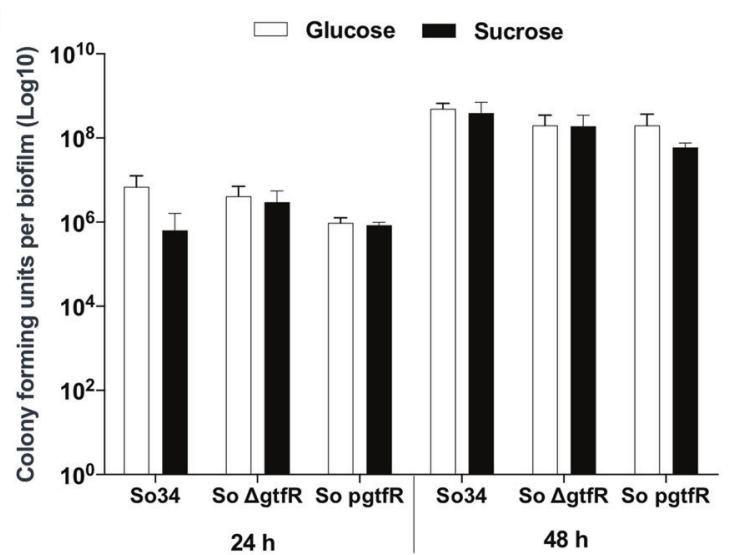

Fig. $1 \mathrm{~S}$. oralis biofilms growing for 24 or $48 \mathrm{~h}$ on polystyrene. Biofilms of wild-type (So34), gtfR mutant (So $\Delta \mathrm{gtfR}$ ) or complemented (So pgtfR) strains were grown in RPMI, $10 \% \mathrm{FBS}, 10 \%$ BHI media supplemented with $1 \%$ sucrose or $1 \%$ glucose. a $X-Y$ isosurfaces (top panel) and three-dimensional reconstructions (bottom panel) of representative confocal laser scanning microscopy images of biofilms. S. oralis (blue) was visualized after fluorescence in situ hybridization with a Streptococcus-specific probe conjugated to Alexa 405. Alexa Fluor 647-labeled dextran conjugate probe (red) was used to stain biofilm matrix (glucans). Scale bars, $50 \mu \mathrm{m}$ ( $X-Y$ isosurfaces)
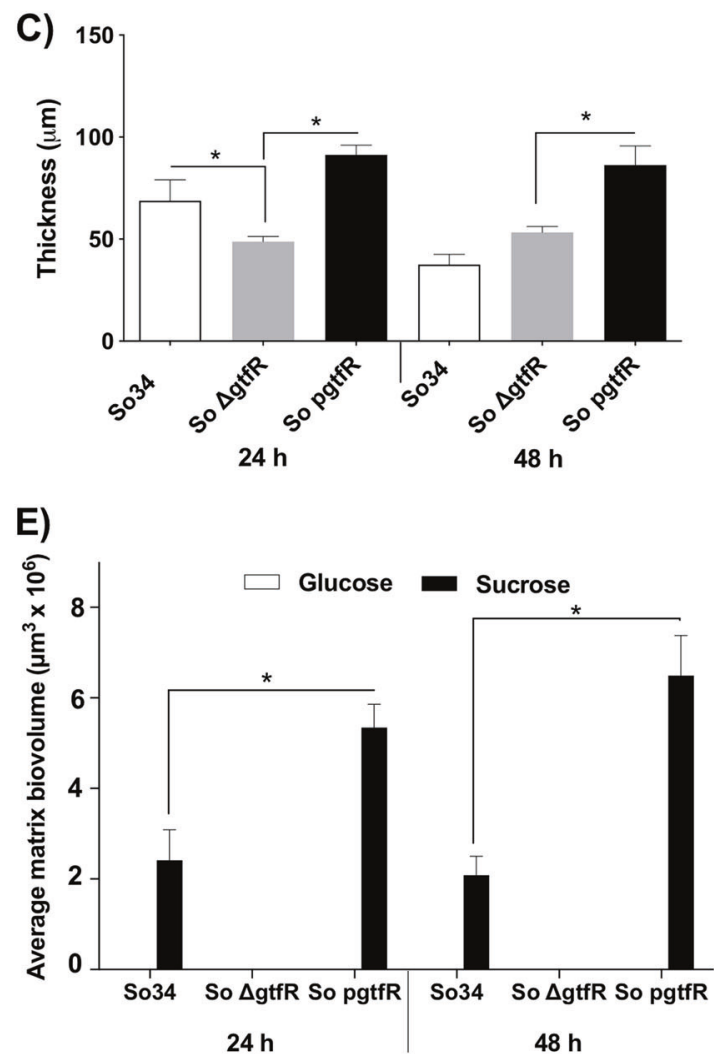

and $70 \mu \mathrm{m}$ (three-dimensional reconstructions). b Average total biovolumes (in $\mu \mathrm{m}^{3}$ ) for 24 and $48 \mathrm{~h}$ biofilms exposed to glucose (white bars) or sucrose (black bars) shown in (a) above. Biovolumes were measured in two different confocal laser scanning microscopy image stacks from two independent experiments. c Average biofilm thickness (in $\mu \mathrm{m}$ ) in biofilms growing with $1 \%$ sucrose for 24 and $48 \mathrm{~h}$. d Average $S$. oralis colony-forming units (CFU) in logarithmic scale for each 24 and $48 \mathrm{~h}$ biofilm. e Average matrix ( $\alpha$-glucan) biovolumes (in $\mu^{3}$ ) for 24 and $48 \mathrm{~h}$ biofilms. ${ }^{*} p<0.05$, using the Bonferroni $t$-test. The error bars indicate standard deviations. 
A)

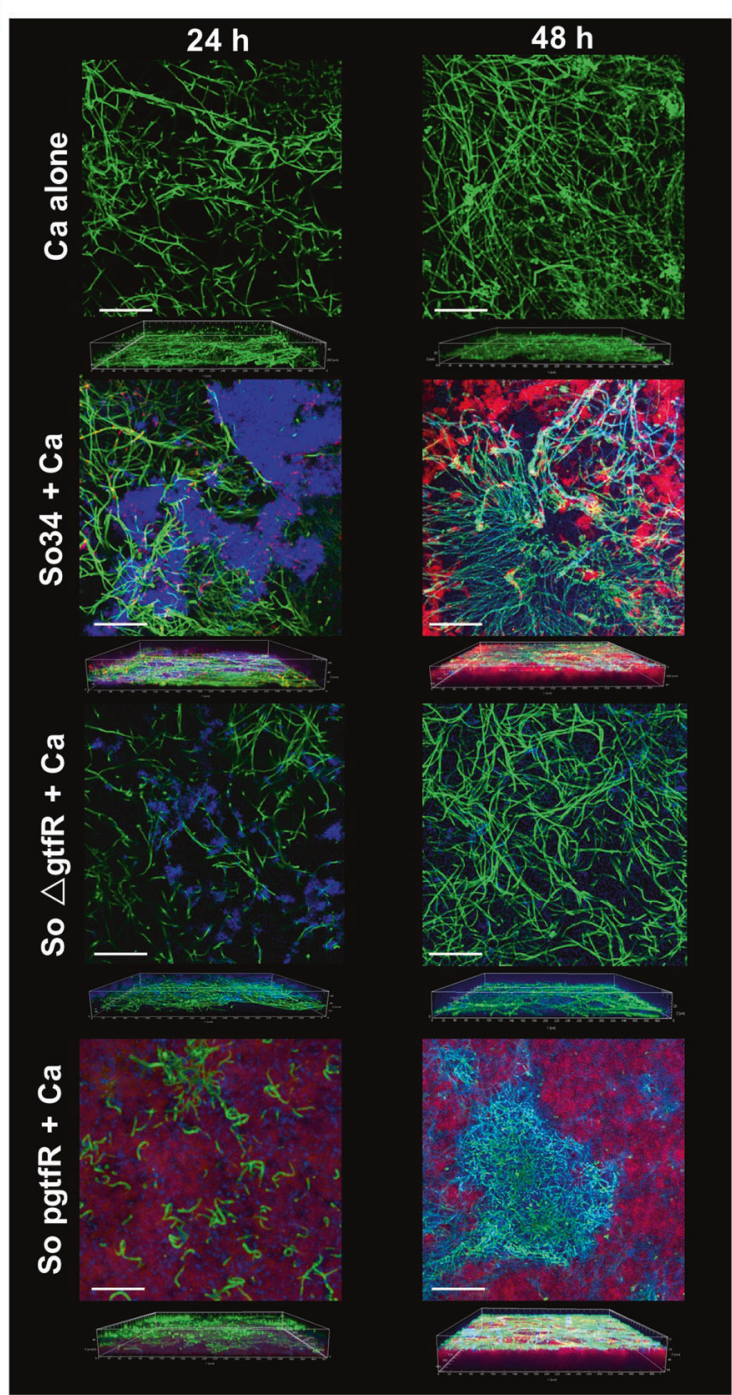

E)

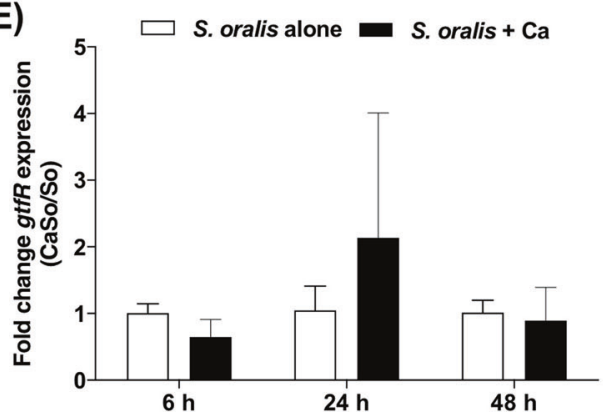

Fig. 2 Twenty-four and forty-eight hour biofilms of C. albicans (Ca) alone or with wild-type $S$. oralis (So34), gtfR mutant (So $\Delta \mathrm{gtfR}$ ) or complemented (So pgtfR) strains. Biofilms were grown on polystyrene surfaces in RPMI, $10 \%$ FBS, $10 \%$ BHI media supplemented with $1 \%$ sucrose. a $X-Y$ isosurfaces (top panel) and three-dimensional reconstructions (bottom panel) of representative confocal laser scanning microscopy images of biofilms. Please note overlap of green and red signals shown in yellow, suggesting close physical proximity between the two organisms. Scale bars $50 \mu \mathrm{m}$. b Average total biofilm
B)

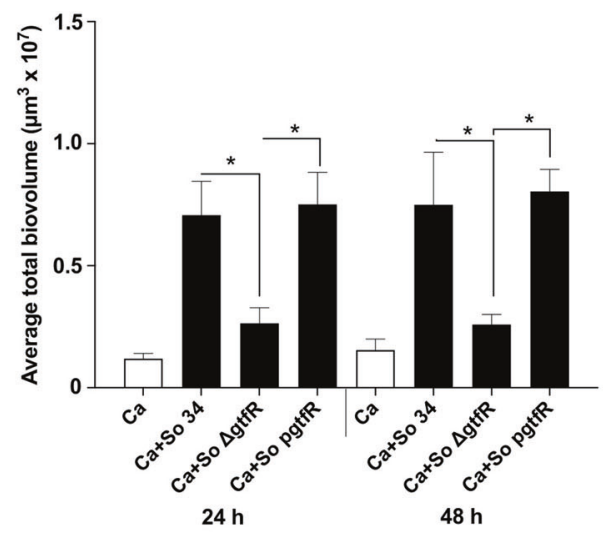

C)

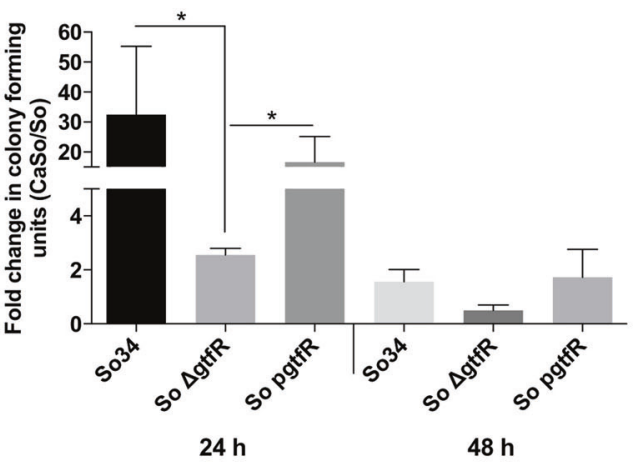

D)

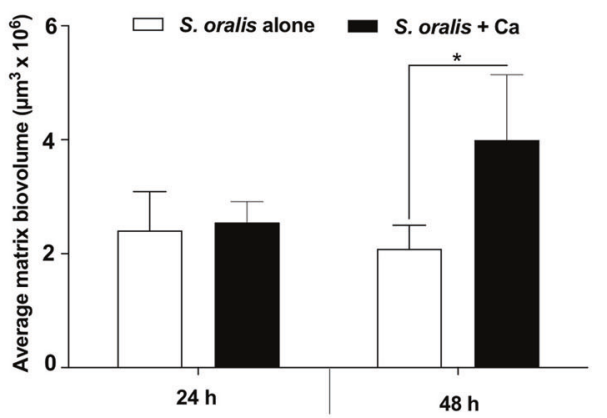

F)

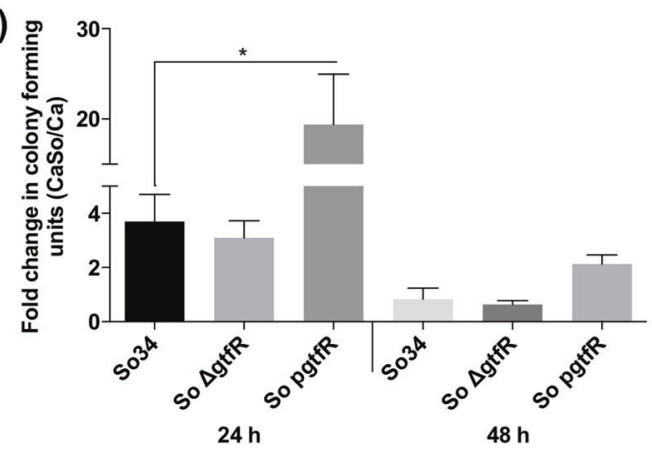

biovolume (in $\mu^{3}$ ) for 24 and $48 \mathrm{~h}$ biofilms. c $S$. oralis CFU counts expressed as fold of mixed over single biofilms. d Average matrix biovolumes (in $\mu^{3}$ ). e Relative expression of $g t f R$ gene levels assessed by RT-qPCR. Results represent mean fold change gene expression in C. albicans with $S$. oralis (CaSo, black bars) over S. oralis (So, white bars) biofilms alone, in three independent experiments. f $C$. albicans CFU counts expressed as fold of mixed biofilms with each of the three S. oralis strains $(\mathrm{CaSo})$ over single biofilms $(\mathrm{Ca}) . * p<0.05$, using the Bonferroni $t$-test. The error bars indicate standard deviations. 
complemented strain (Fig. 2f). We previously reported that in mixed mucosal biofilms $S$. oralis activates the Efgl filamentation pathway and increases hyphae-associated gene expression in $C$. albicans [23]. We thus tested whether $g t f R$ influences these interactions by comparing the wild-type and mutant strains. Both the wild-type and $\Delta g t f R$ strains upregulated Candida genes in this pathway after $6 \mathrm{~h}$ of interaction however, there was no difference between the two strains (Fig. S2A).

\section{The type of substratum affects $g t f R$-associated biofilm phenotypes}

Titanium is a commonly used biomaterial that supports mixed species biofilm growth [34]. We thus tested the gtfRassociated biofilm phenotype on this substratum. On titanium surfaces wild-type $S$. oralis formed thick biofilms enmeshed in $\alpha$-glucan-rich matrix (Fig. 3a). Deletion of $g t f R$ reduced the biofilm density and total biovolume, without a significant effect on bacterial biovolume (Fig. 3a, b) or CFU counts (data not shown) similar to the polystyrene phenotype. Confocal imaging showed sparsely distributed single biofilms of $C$. albicans on titanium surfaces (Fig. 3a).

On titanium we noted a robust mixed biofilm growth of the wild-type and complemented $S$. oralis strains with C. albicans (Fig. 3a), with significantly greater total biovolume compared with biofilms with the gtfR mutant (Fig. 3b). In biofilms with the wild-type and complemented strains we also noted a pronounced bacterial co-aggregation, with $C$. albicans mostly forming a layer over the streptococcal aggregates (Fig. 3a). There was a lower (albeit not statistically significant) bacterial biovolume and a significant reduction in bacterial viable counts between wild-type and mutant strains (Fig. 3b, c). In biofilms with wild-type $S$. oralis the fungal biovolume was significantly increased compared with single $C$. albicans biofilms (Fig. 3b). In contrast to polystyrene surface biofilms, fungal biomass on titanium was significantly higher in mixed biofilms with the wild-type $S$. oralis strain compared with the gtfR mutant, as reflected by biovolume and viable count estimates (Fig. 3b, d). These results suggest that in mixed biofilms GtfR promotes fungal accretion on titanium surfaces.

As seen in biofilms forming on polystyrene surfaces, mixed species biofilms of the wild-type strain with C. albicans had increased $\alpha$-glucan matrix biovolume compared with monospecies biofilms (Fig. 3e). However, there were significantly lower amounts of matrix on titanium compared with polystyrene (Fig. S3). In $24 \mathrm{~h}$ mixed biofilms we also found an increase in $g t f R$ gene expression compared with monospecies biofilms (Fig. 3f). In summary these data suggest that in mixed biofilms growing on titanium $g t f R$ has a positive influence on both fungal and bacterial biomass. These results also show that on titanium surfaces $C$. albicans enhances both streptococcal cell numbers and gtfR expression, which could contribute to an increase in $\alpha$-glucan-rich matrix synthesis in late stages.

To extend these findings we next investigated the influence of $g t f R$ in biofilms growing on an organotypic mucosa. Because $S$. oralis 34 wild-type strain alone does not form a biofilm on this surface [19] we focused on the influence of $g t f R$ in mixed biofilms with $C$. albicans. Both $\Delta g t f R$ and wild-type strains formed a mixed biofilm with C. albicans on the mucosal surface (Fig. 4a). Similar to abiotic surfaces, there was a significantly greater bacterial biofilm growth in the wild type (and complemented, data not shown) compared with the $\Delta g t f R$ strain as assessed by viable counts (Fig. 4b), showing that $g t f R$ promotes $S$. oralis biofilm growth with $C$. albicans. However, $\alpha$ glucan matrix staining and biovolume estimates showed lower amounts of matrix produced by the wild-type strain in mixed biofilms on mucosa compared with other surfaces (Figs. 4c and S3).

Unlike biofilms growing on titanium, the fungal biomass on mucosal surfaces was not significantly affected by $g t f R$ gene deletion, as shown by viable counts (Fig. 4b). Along the same lines $C$. albicans genes related to hyphal transformation were not influenced by $g t f R$ gene deletion (Fig. S2B). Based on these findings we conclude that GtfR can enhance $C$. albicans-S. oralis mixed biofilm formation on mucosal surfaces by a positive effect on bacterial biomass, possibly by enhancing streptococcal cell-cell coaggregation interactions, even though the $\alpha$-glucan matrix was not a major component of these biofilms.

\section{GtfR-derived glucans promote C. albicans biofilm growth on abiotic surfaces}

Prompted by the positive influence of $S$. oralis gtfR on C. albicans biofilm growth on abiotic surfaces, we further explored the mechanism of this interaction. C. albicans adhered poorly when inoculated in $1 \%$ sucrose-supplemented media on titanium surfaces, compared with polystyrene (Fig. S4A). We then hypothesized that $\alpha$-glucans may promote adhesion of $C$. albicans which leads to greater biofilm growth. We first tested the effect of dextran, a soluble form of $\alpha$-glucan, on adhesion and biofilm growth of $C$. albicans. Dextran coating improved adhesion of $C$. albicans to polystyrene (shown by reduction in nonadherent cells, Fig. S4B); however, this did not significantly affect $C$. albicans biofilm biomass as assessed by viable cell counts (Fig. S4C).

We then tested whether native $\alpha$-glucans synthesized by wild-type $S$. oralis increase $C$. albicans biofilm accretion by a two-pronged approach. First we inoculated $C$. albicans yeast cells on preformed biofilms of the $\Delta g t f R$ or wild-type $S$. oralis strains (on polystyrene or titanium) and compared fungal biovolumes after 1 or $16 \mathrm{~h}$ of incubation. In the absence of 
A)
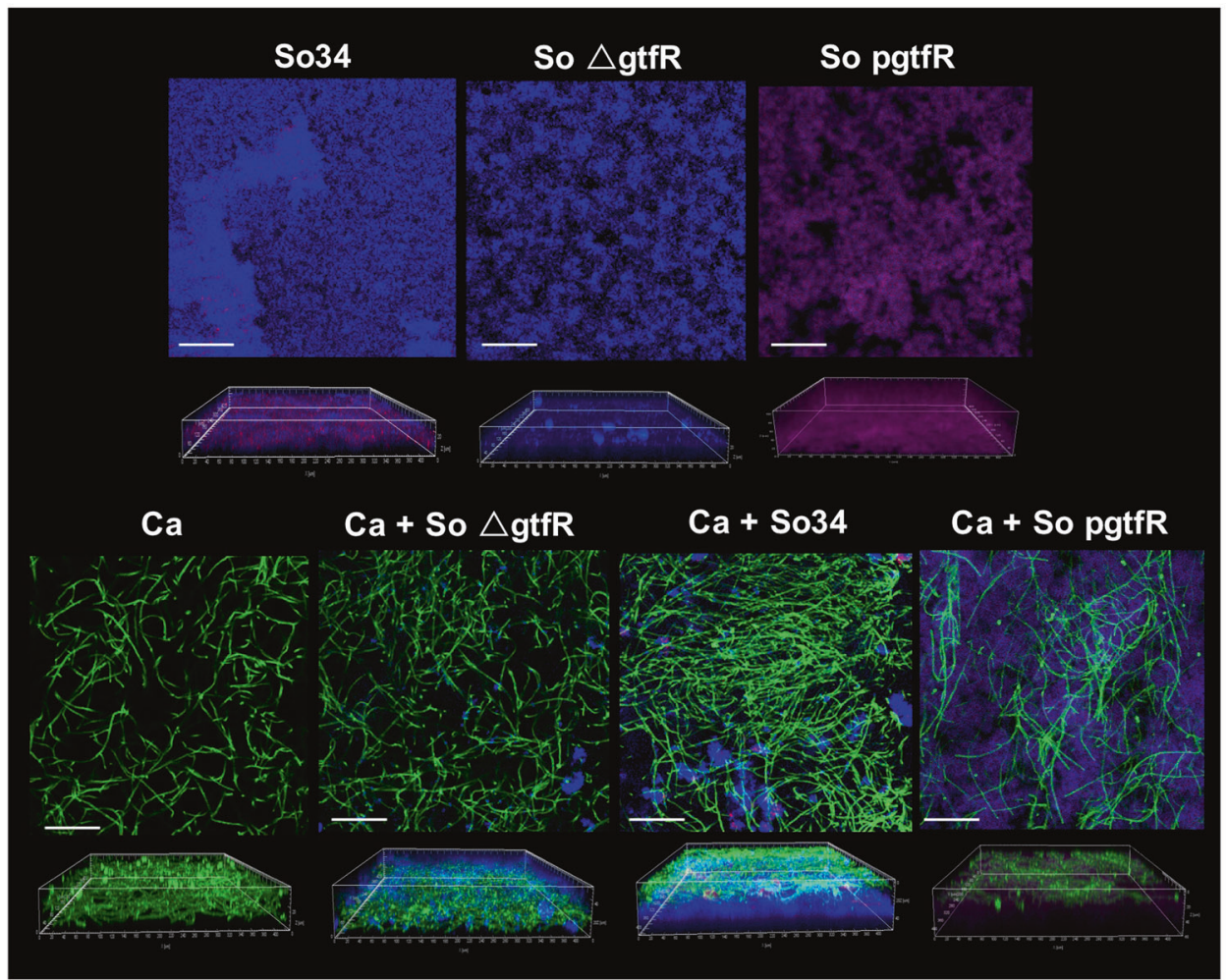

B)

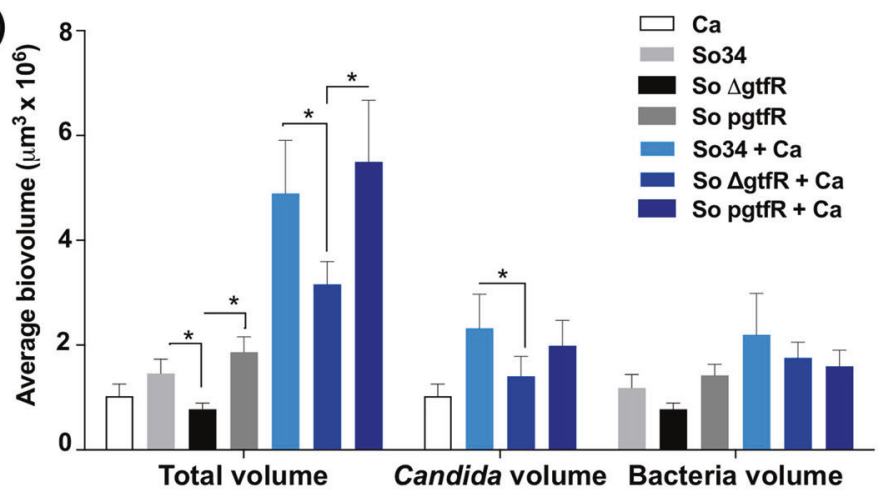

C)

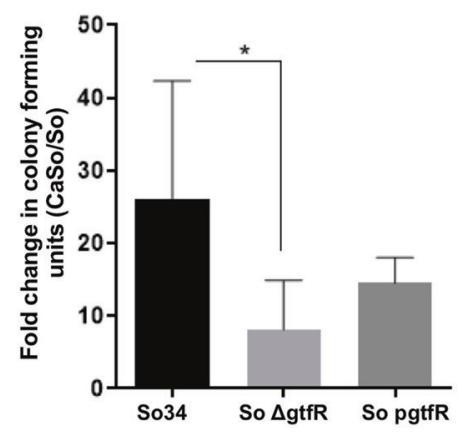

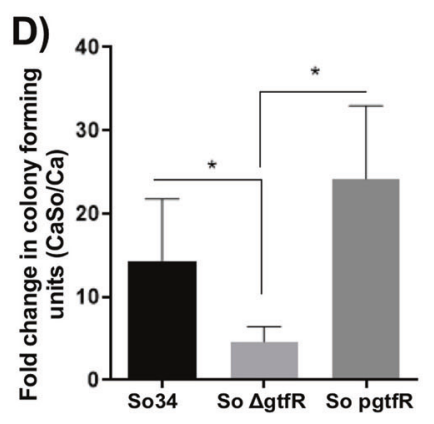

E)

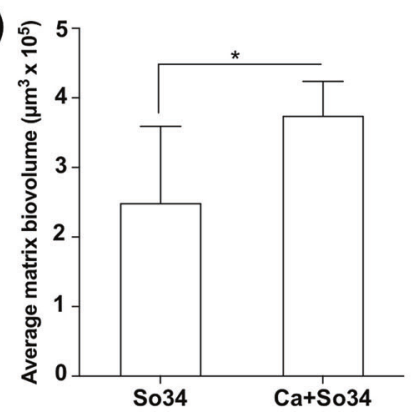

F)

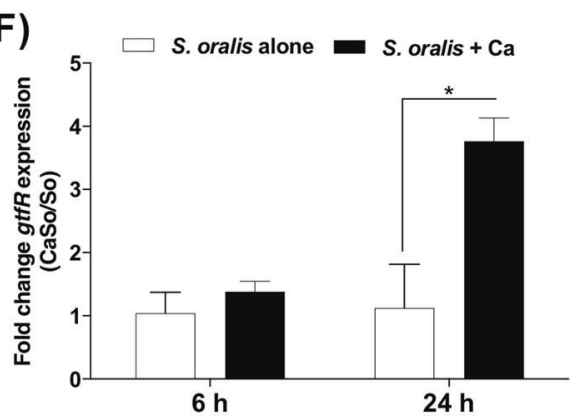

sucrose, C. albicans biovolumes were not significantly different when fungi were inoculated directly on polystyrene or titanium, compared with inoculation onto a preformed $\Delta g t f R$ or WT strain biofilm (Fig. 5). However, a significant increase in fungal biovolumes was noted when $C$. albicans was inoculated on a preformed biofilm of wild-type $S$. oralis grown with sucrose and results were similar on both titanium and polystyrene surfaces (Fig. 5a-d). These results suggested that GtfR-synthesized $\alpha$-glucans were responsible for increased C. albicans accretion.

To strengthen these observations, in a second approach we explored whether $\alpha$-glucans synthesized by the purified 
Fig. 3 Biofilms growing on titanium surfaces for $24 \mathrm{~h}$ with $1 \%$ sucrose. S. oralis (So) (wild-type So34, $\Delta g t f R$ mutant, or complemented pgtfR strains) and $C$. albicans $(\mathrm{Ca})$ growing alone or in combination. a $X-Y$ isosurfaces (top panel) and three-dimensional reconstructions (bottom panel) of representative confocal laser scanning microscopy images of biofilms. Organisms and $\alpha$-glucan-rich matrix were visualized by staining as above. Scale bars, $50 \mu \mathrm{m}(X-Y$ isosurfaces) and $70 \mu \mathrm{m}$ (three-dimensional reconstructions). b Average total, Candida or bacterial biovolumes (in $\mu^{3}{ }^{3}$ ). Biovolumes were measured in two different confocal laser scanning microscopy image stacks from two independent experiments. c $S$. oralis $\mathrm{CFU}$ counts shown as mean fold of mixed biofilms over single biofilms in three experiments. d Candida CFU counts shown as fold of mixed biofilms over single biofilms. e Average matrix ( $\alpha$-glucans) biovolumes (in $\mu^{3}$ ) on $S$. oralis (WT) alone biofilms and C. albicans-streptococci mixed species biofilm. f Relative expression levels of $g t f R$ gene in $S$. oralis strain 34 were analyzed by RT-qPCR. Results represent mean fold change gene expression in $C$. albicans with $S$. oralis (CaSo) over $S$. oralis (So) alone biofilms in independent experiments. $* p<0.05$, using the Bonferroni $t$-test. The error bars indicate standard deviations.

native enzyme from wild-type S. oralis 34 (Fig. S4D) promote fungal adhesion on abiotic surfaces. We first demonstrated that when polystyrene surfaces were coated with the purified enzyme, adding $1 \%$ sucrose led to synthesis of $\alpha$-glucan which could be visualized coating the surface by confocal microscopy (Fig. S4E). When C. albicans was inoculated on $\alpha$-glucan-coated surfaces there was significantly higher adhesion compared with untreated surfaces or surfaces that were coated with GtfR in the absence of sucrose (Fig. 6a, b). These results further suggest that $\alpha$ glucan is responsible for the increase in $C$. albicans initial attachment and biofilm accretion on abiotic surfaces.

\section{GtfR binding to C. albicans does not play a role in interspecies interactions}

Gtf proteins have a catalytic active site domain necessary for hydrolysis of sucrose, and a series of direct YG repeats in the carboxyl terminus thought to function in glucan binding. We next questioned whether the GBD of the GtfR protein could interact directly with $C$. albicans possibly leading to increased accretion or interspecies co-aggregation interactions in mixed biofilms. After optimizing the protein concentration needed to coat 96 well plates (Fig. S5A) we first tested the ability of rGBD-coated wells to bind biotinconjugated dextran. All tested concentrations of dextran bound to the rGBD-coated wells (Fig. S5B). rGBD bound
A)

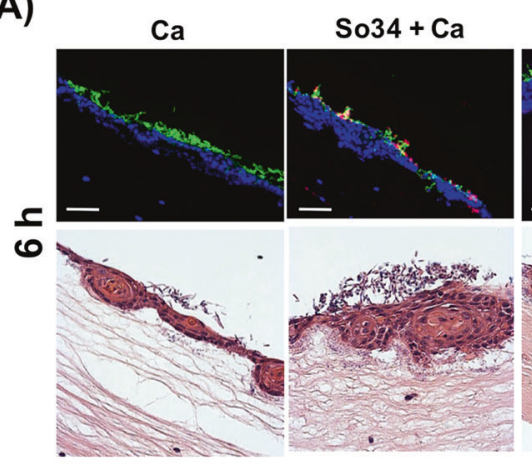

So $\triangle \mathrm{gtfR}+\mathrm{Ca}$

B)

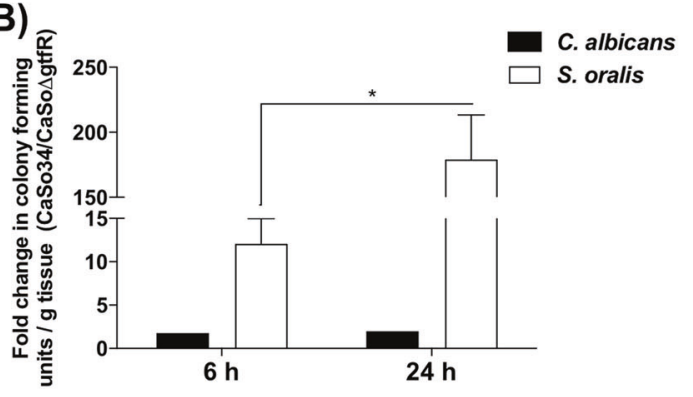

Fig. 4 Biofilms of $C$. albicans alone or in combination with $S$. oralis (WT and $\Delta g t f R$ strains) growing on organotypic mucosal surfaces for 6 or 24 h. C. albicans (green) was visualized after staining with an FITC-conjugated anti-Candida antibody. S. oralis (red) was visualized after fluorescence in situ hybridization with a Streptococcus-specific probe conjugated to Alexa 546. a Tissue sections of mucosal biofilms with organisms stained as above, and mucosal cell nuclei counterstained with the nucleic acid stain Hoechst 33258 (blue, top panel). Corresponding haematoxylin and eosin-stained tissue sections are

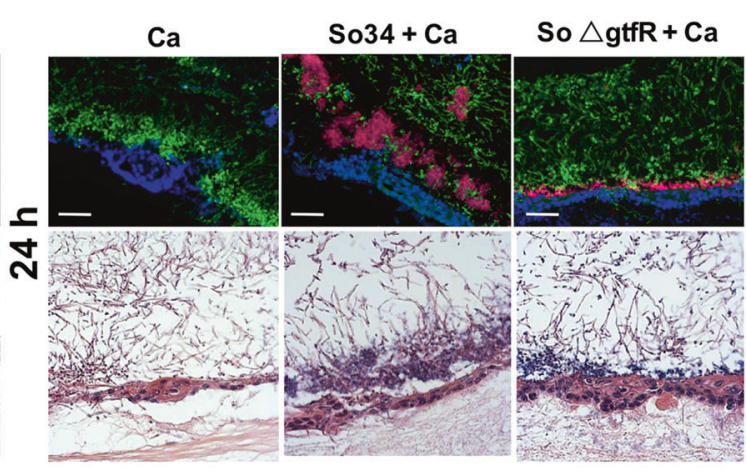

C)

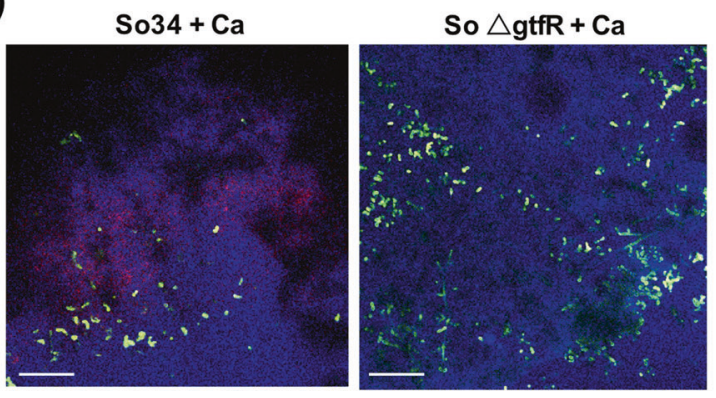

shown in the bottom panels. Scale bars $20 \mu \mathrm{m}$. b C. albicans (black bars) and $S$. oralis 34 (white bars) CFU counts expressed as fold of Candida and wild-type $S$. oralis mixed biofilms (CaSo34) over Candida and $\Delta g t f R$ mutant mixed biofilms ( $\mathrm{CaSo} \Delta \mathrm{gtfR})$. c $X-Y$ isosurfaces of representative confocal laser scanning microscopy images of mixed $24 \mathrm{~h}$ biofilms (green, Candida, blue, $S$. oralis) showing $\alpha$ glucans (biofilm matrix) stained with Alexa Fluor 647-labeled dextran conjugate probe (red). Scale bars $50 \mu \mathrm{m}$. ${ }^{*} p<0.05$, using the Bonferroni $t$-test. The error bars indicate standard deviation. 
A)
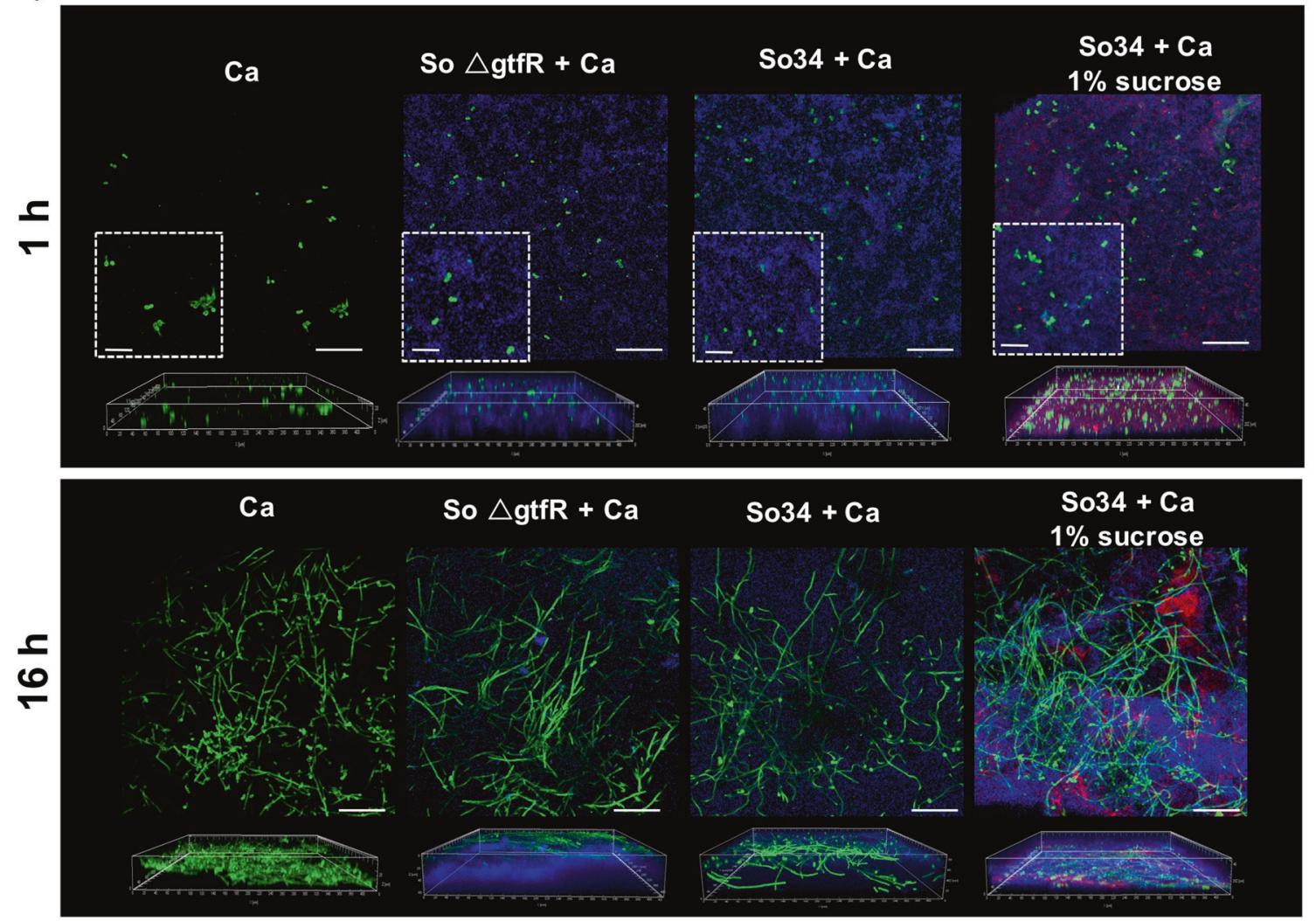

B)

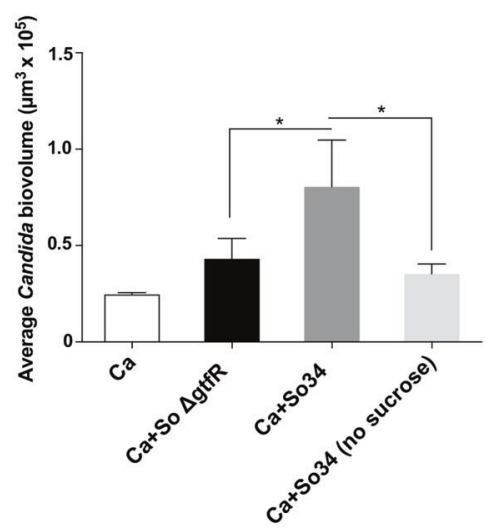

Fig. 5 C. albicans interactions with preformed $S$. oralis biofilms. $S$. oralis biofilms were grown for $24 \mathrm{~h}$ using wild-type (So34) or $\Delta g t f R$ strains; media were supplemented with $1 \%$ sucrose or no carbohydrate. C. albicans was then added and incubated for 1 or $16 \mathrm{~h}$. Unattached cells were washed and biofilms were stained. a $X-Y$ isosurfaces (top panel) and three-dimensional reconstructions (bottom panel) of representative confocal laser scanning microscopy images of biofilms. C. albicans (green) was visualized after staining with an FITCconjugated anti-Candida antibody. S. oralis (blue) was visualized after fluorescence in situ hybridization with a Streptococcus-specific probe

to the surface of only a small percentage of yeast cells (5-7\%) as shown by immunofluorescence and FACS analyses (Fig. S6A). Increasing the concentrations of rGBD did
D)

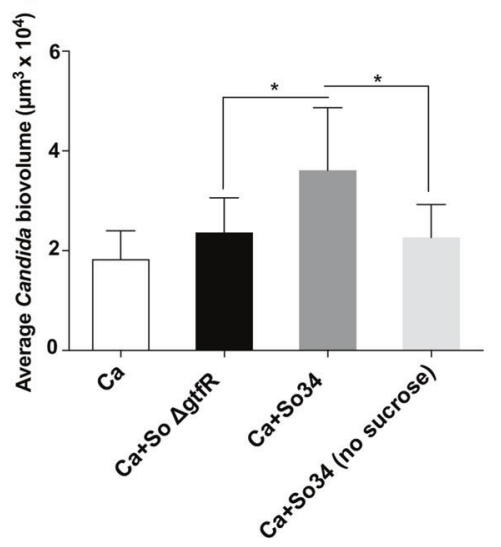

conjugated to Alexa 405. Alexa Fluor 647-labeled dextran conjugate probe (red) was used to label biofilm matrix ( $\alpha$-glucans). Scale bars, 50 or $20 \mu \mathrm{m}(X-Y$ isosurfaces) and $70 \mu \mathrm{m}$ (three-dimensional reconstructions). Average Candida biovolumes (in $\mu^{3}$ ) after $1 \mathrm{~h}$ adhesion on $S$. oralis biofilms formed on polystyrene for $1 \mathrm{~h}(\mathbf{b})$ or $16 \mathrm{~h}(\mathbf{c})$. Similar experiments were performed on titanium surfaces and C. albicans biovolumes were quantified after $16 \mathrm{~h}(\mathbf{d}) .{ }^{*} p<0.05 \mathrm{using}$ the Bonferroni $t$-test. The error bars indicate standard deviations in triplicate experiments.

not increase protein binding on $C$. albicans surface in the yeast form (Fig. S6B), or in early or late C. albicans biofilms (data not shown). We next examined whether purified 
A)

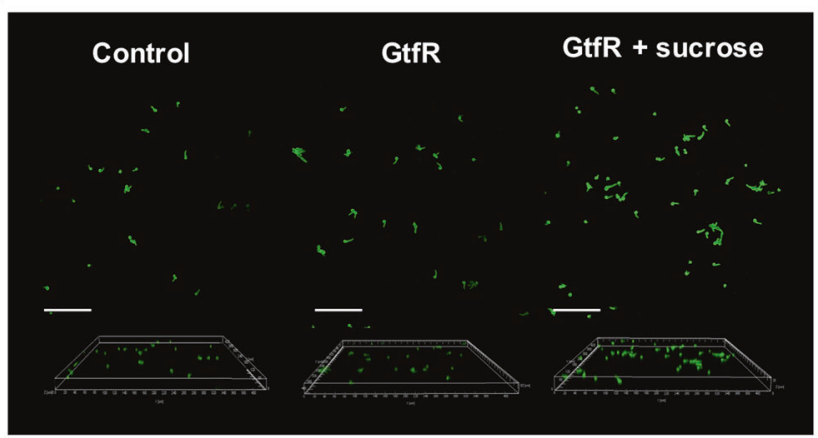

B)

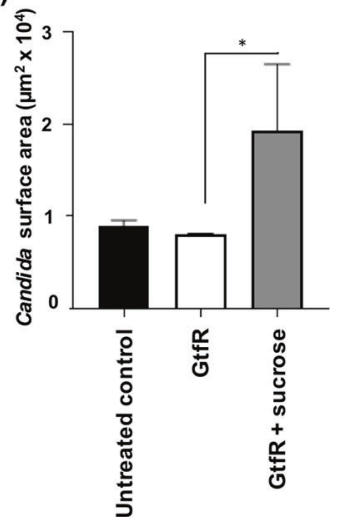

Fig. 6 C. albicans adhesion on polystyrene surfaces. a Representative confocal images of $C$. albicans. Surfaces were precoated with purified GtfR $(1 \mu \mathrm{g} / \mathrm{ml})$ in the presence or absence of $1 \%$ sucrose for 1 $\mathrm{h}$ and $C$. albicans was inoculated after washing excess sucrose. C. albicans (green) was visualized $1 \mathrm{~h}$ post inoculation by staining

native GtfR can bind to a preformed $24 \mathrm{~h}$ fungal biofilm and synthesize $\alpha$-glucans on the surface of fungi after overnight incubation with $1 \%$ sucrose. We could not detect Alexa Fluor 647-labeled $\alpha$-glucans on the surface of fungal cells (data not shown). We conclude that GtfR binding to fungal cells does not play a major role in the biofilm interactions of C. albicans and S. oralis.

\section{S. oralis mucosal biomass is enhanced by $g t f R$ in vivo}

We next tested the role of $g t f R$ in S. oralis mucosal biofilms in vivo. To unravel sucrose-dependent effects of GtfR mice received 5\% sucrose in their drinking water [35]. In these experiments the wild-type mucosal burdens, as assessed by a strain-specific qPCR, were significantly higher than the $\Delta g t f R$ mutant in both single and mixed infections (Fig. 7a), and these results were confirmed by viable counts (data not shown). On the other hand the fungal biomass was not significantly affected by either the wild-type or mutant strains (Fig. 7b). In mice infected with $C$. albicans fungal cells were primarily in the yeast form, and in mixed infection with the wild-type $S$. oralis, yeast were interspersed within a matrix-like material occupying the space between the filiform papillae. This material was less abundant in mice infected with wild-type $S$. oralis only (Fig. 7c).

Sucrose increased the mucosal burdens of wild-type $S$. oralis (Fig. S7A), but not the $\Delta g t f R$ mutant (data not shown). As published previously [18], C. albicans coinfection increased $S$. oralis burdens in mice not receiving sucrose. However, there was a significant drop in $S$. oralis burdens in co-infected mice receiving sucrose (Fig. S7A). Sucrose negatively influenced $C$. albicans burdens in both with an FITC-conjugated anti-Candida antibody. b Average surface area covered by Candida cells as quantified by Image $\mathbf{J}$ analysis of three microscopic fields in each of two independent experiments. $* p<0.05$ using the Bonferroni $t$-test. The error bars indicate standard deviations.

single and mixed infection models (Fig. S7B). These results suggested that the effect of sucrose on $S$. oralis burdens in co-infected mice was a consequence of the reduction in Candida burdens. We next hypothesized that the reduction in Candida burdens could be due to overgrowth of antagonistic bacteria promoted by sucrose. To test this hypothesis we assessed the impact of sucrose on the mucosal bacteriome of Candida-infected mice via 16S rRNA gene sequencing (Fig. 8). Nonmetric multidimensional scaling (NMS) analysis of Bray-Curtis dissimilarities showed that the bacterial microbiome composition was distinct in $\mathrm{Can}$ dida-infected mice that received sucrose compared with ones that did not (Fig. 8a). Analysis of the most prevalent bacterial OTUs (minimum $1 \%$ of the reads in at least one sample/group) revealed distinct genus level differences between the two groups (Fig. 8b). We found that sucrose caused a statistically significant increase in the relative abundance of endogenous streptococci, whereas lactobacilli also increased but this did not reach statistical significance. On the other hand there was a statistically significant decrease in enterococci in Candida-infected mice receiving sucrose. (Fig. 8c).

\section{Discussion}

Previous studies showed that $C$. albicans and $S$. oralis have a mutualistic relationship in the biofilm growth state which promotes fungal virulence [22, 23]. In this work we showed that $g t f R$ promotes sessile growth of $S$. oralis on all tested surfaces in vitro and in vivo, when sucrose is available. However, in mixed biofilms with $C$. albicans the role of $g t f R$ is modulated by the type of biofilm substratum and the mucosal environment. This may be due to the different 
A)

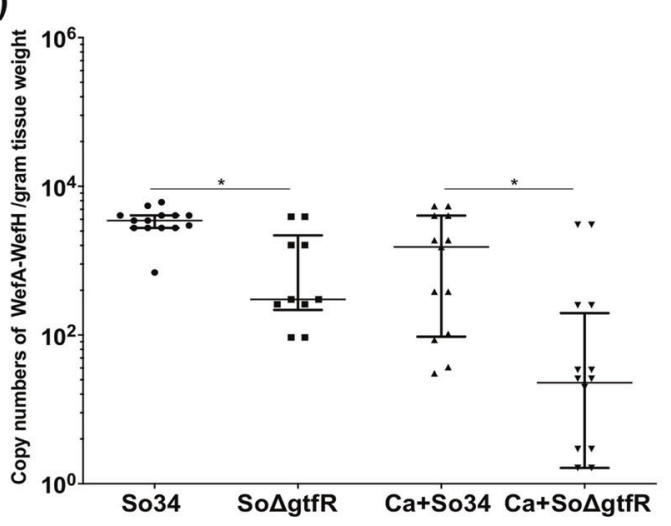

B)

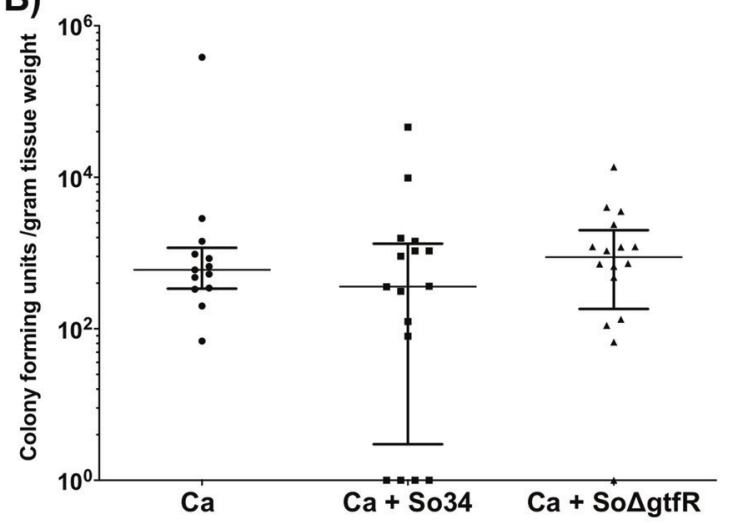

C)

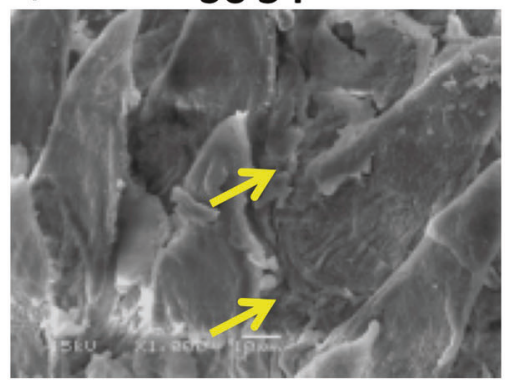

$\mathrm{Ca}+\mathrm{So} 34$
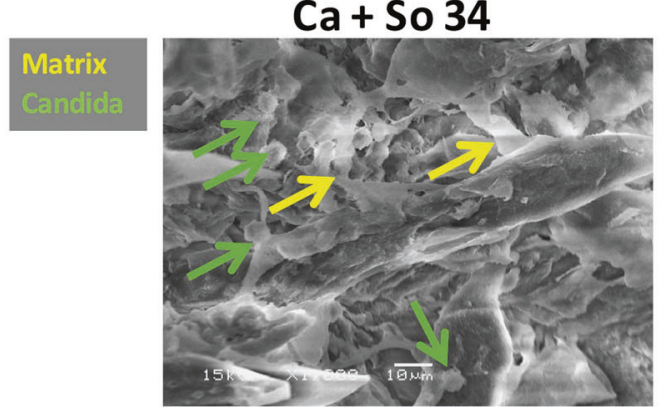

Fig. 7 Role of $\boldsymbol{g t f R}$ in mucosal biofilms in vivo. Mice were inoculated with $S$. oralis wild-type (So34) or $\Delta g t f R$ strains, with or without $C$. albicans $(\mathrm{Ca})$, and tongues were excised 5 days post inoculation at necropsy. a $S$. oralis mucosal burdens analyzed by qPCR using DNA extracted from tongues, and primers specific for the $S$. oralis 34 wefA$\mathrm{H}$ gene. Cell (gene copy) numbers were calculated according to standard curves using known amounts of $S$. oralis 34 or $\Delta g t f R$ strain gDNA, and normalized over tissue weight. b $C$. albicans mucosal

amounts of $\alpha$-glucan matrix on different substrata, which is more abundant on abiotic compared with mucosal surfaces. On abiotic surfaces $C$. albicans co-inoculation with $S$. oralis increased the amount of $\alpha$-glucan matrix, by increasing bacterial cell numbers or $g t f R$ gene expression. We showed that on these surfaces $C$. albicans adhesion and growth is promoted by the presence of preformed GtfR-synthesized $\alpha$ glucans. Our studies are the first to provide experimental evidence that $\alpha$-glucans synthesized by GtfR are important in cross-kingdom interactions between $C$. albicans and S. oralis favoring biofilm growth.
So $\Delta g t f R$

C. albicans

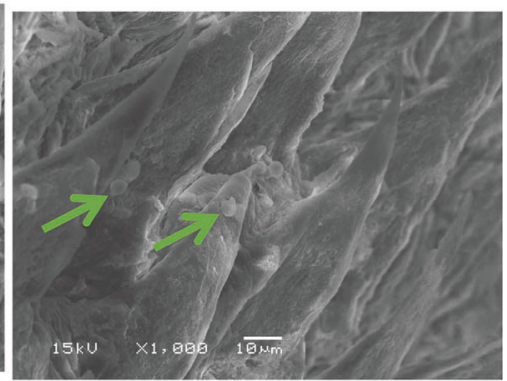

Ca + So $\Delta$ gtfR

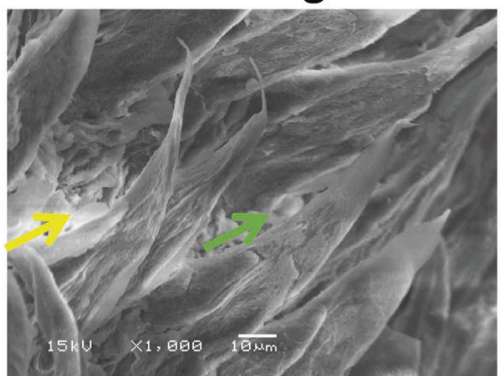

burdens as assessed by viable counts in tongue homogenates, normalized by tissue weight. Results of two independent mouse experiments, with 6-8 animals/group are shown. c Representative SEM images of biofilms forming on the tongue surface. Yellow arrows indicate the matrix-like material filling the spaces between filiform papillae in mice infected with wild-type $S$. oralis and $C$. albicans. Green arrows indicate yeast cells. $* p<0.05$ using the Bonferroni $t$-test.

We found that the mucosal environment in vivo modulates sucrose-dependent interactions of the two organisms. One important finding was that sucrose reduces $C$. albicans mucosal burdens, in both single and mixed inoculation models. High glucose availability resulting from sucrose hydrolysis may repress $C$. albicans morphogenesis [36] and promote yeast dispersion [37]. This may explain the predominant yeast form on the tongue surface and the lower fungal burdens in mice receiving sucrose. Lower $C$. albicans burdens combined with reduced hyphae-associated cell wall proteins that enhance inter-kingdom interactions [17], may 
A)

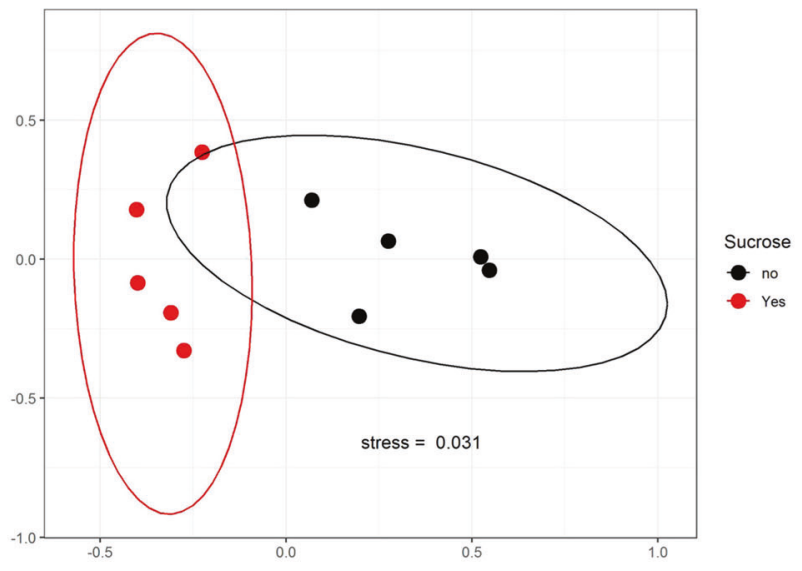

B)

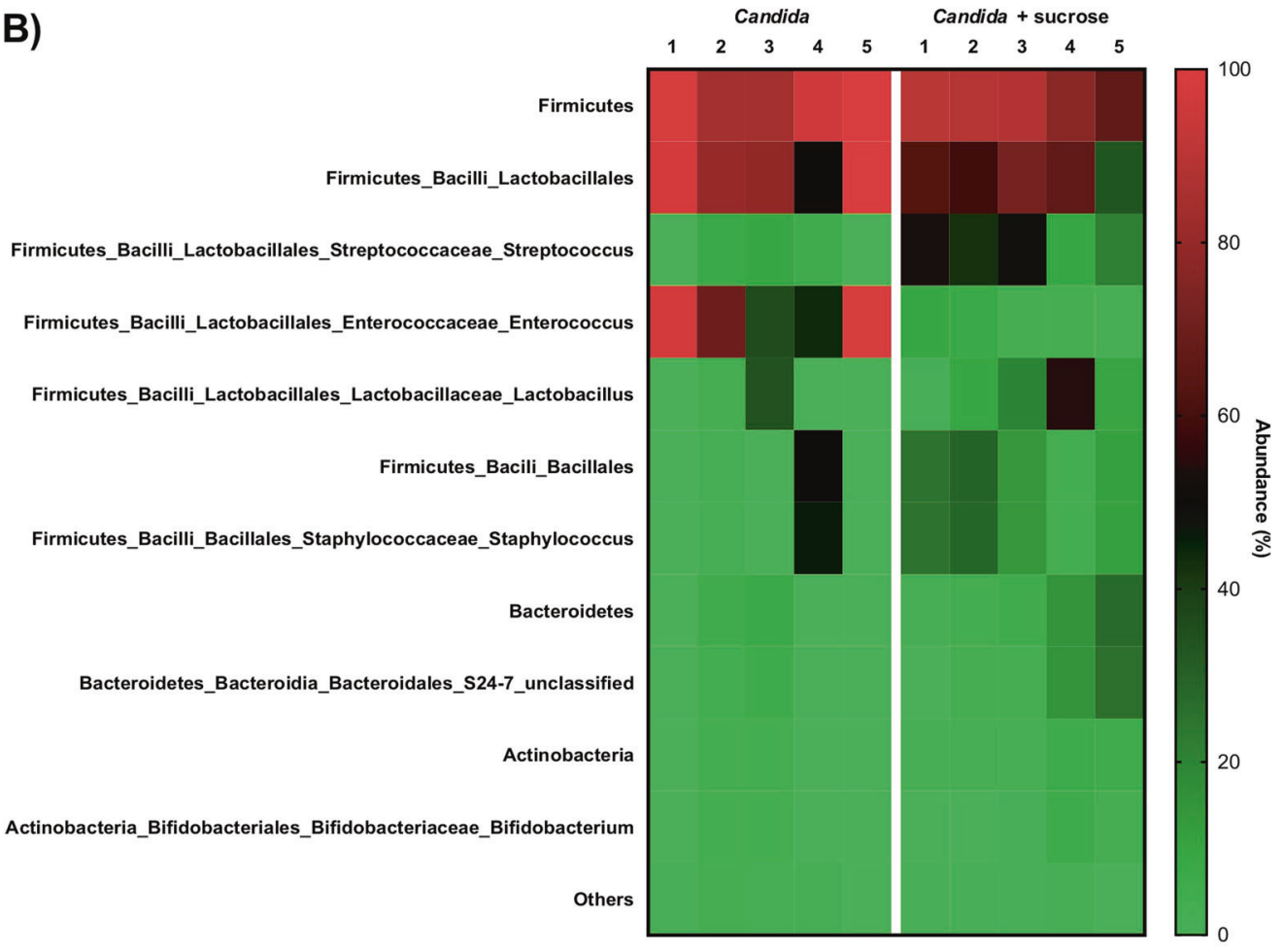

Firmicutes_Bacilli_Lactobacillales_Streptococcaceae_Streptococcus

Firmicutes_Bacilli_Lactobacillales_Enterococcaceae_Enterococcus

Firmicutes_Bacilli_Lactobacillales_Lactobacillaceae_Lactobacillus

Firmicutes_Bacili_Bacillales

Firmicutes_Bacilli_Bacillales_Staphylococcaceae_Staphylococcus

Bacteroidetes

Bacteroidetes_Bacteroidia_Bacteroidales_S24-7_unclassified

Actinobacteria

Actinobacteria_Bifidobacteriales_Bifidobacteriaceae_Bifidobacterium

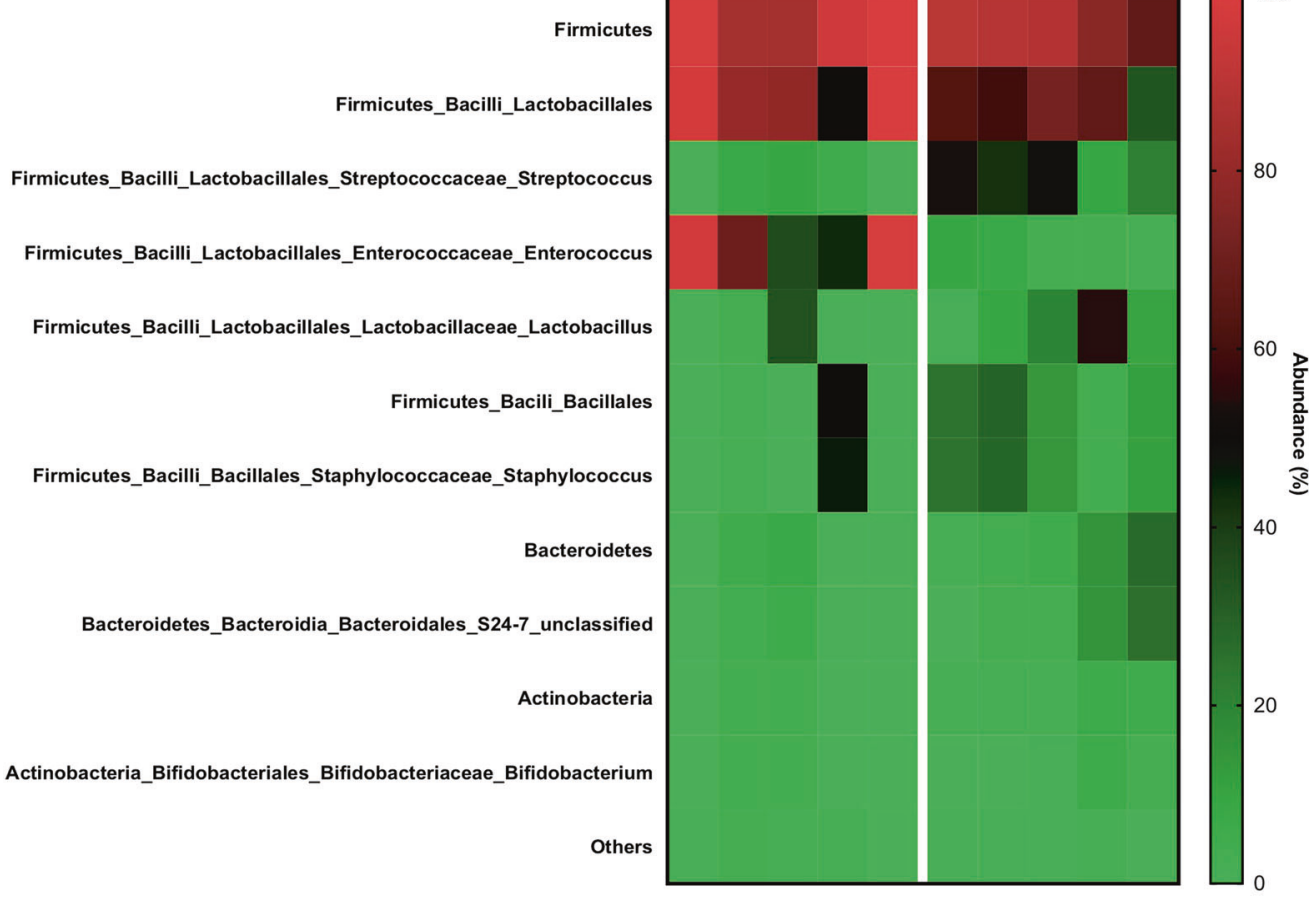

\section{C)}

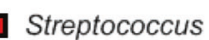

Enterococcus

$\left.{ }^{100}\right]$ [Lactobacillus

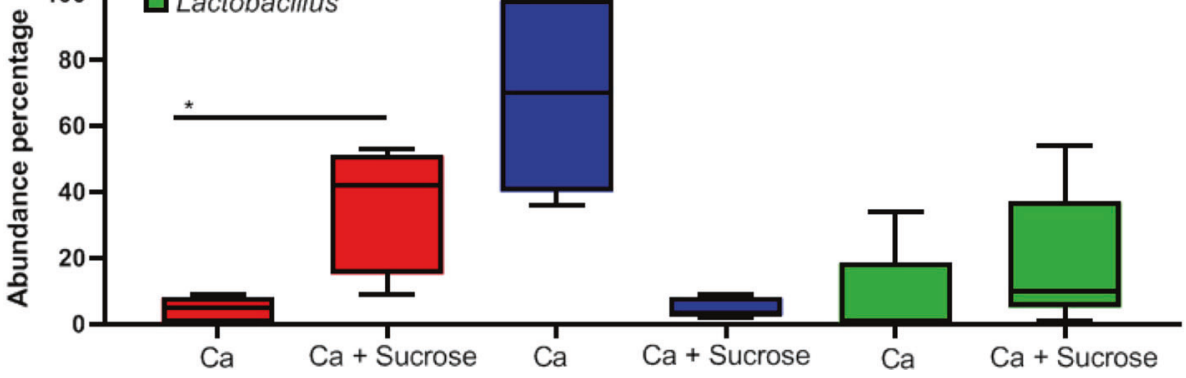

lead to reduction in $S$. oralis burdens in co-infected mice receiving sucrose. Exposure to a high sucrose diet changes the composition of the endogenous microbial communities in the alimentary tract of mice [38]. Such changes may involve overgrowth of endogenous lactic acid bacteria which curtail C. albicans growth and/or hyphal morphogenesis [39]. 
Fig. 8 Mucosal bacterial microbiome analyses based on highthroughput 16S rRNA gene sequencing. a Beta diversity assessed by nonmetric multidimensional scaling (NMS) based on Bray-Curtis dissimilarities among the treatment groups. Shown are community structures in mice infected with $C$. albicans, in the presence or absence of added sucrose. Results represent bacterial community structure differences at the end of the experimental period (day 5). Communities clustered by type of treatment, indicating a significant effect of sucrose, which explained $52 \%$ of the variability $(p<0.02)$. b Relative abundance of bacterial 16S rRNA gene sequences corresponding to major mucosal genera in mice infected with $C$. albicans, in the presence or absence of added sucrose. c Relative abundance of endogenous Streptococcus, Enterococcus, and Lactobacillus in mice infected with $C$. albicans in the presence or absence of added sucrose, based on 16S rRNA gene sequences. ${ }^{*} p<0.05$ for a $t$-test comparison of the two indicated groups.

Consistent with this, our 16S rRNA gene analyses revealed an increase in abundance of lactic acid bacteria such as streptococci and lactobacilli in Candida-infected mice receiving sucrose. This is consistent with the positive effect of sucrose on exogenously supplied $S$. oralis. In contrast there was a reduction in enterococci in Candida-infected mice receiving sucrose. It is possible that, like lactobacilli, endogenous streptococcal species have antagonistic relationships with $C$. albicans. Of note, $S$. oralis and other human streptococcal species with symbiotic relationships with this fungus are not part of the mouse microbiome $[18,33,40]$. However, we have reported a symbiotic relationship between oral endogenous enterococci and C. albicans in mice [33], consistent with the reduction in the abundance of these organisms in mice receiving sucrose that have reduced $C$. albicans burdens.

The role of $\alpha$-glucans has been mainly explored in relation to $S$. mutans and $C$. albicans and the development of dental caries [40]. S. mutans GtfB is able to bind avidly on mannans located on the outer surface $C$. albicans cell wall [41] and to synthesize extracellular $\alpha$-glucans on the fungal surface [32]. In mixed biofilms forming on hydroxyapatite surfaces, $C$. albicans increases $S$. mutans extracellular biofilm matrix formation by upregulating $g t f B$ and $g t f C$ expression [24, 32]. In our studies $C$. albicans induced an upregulation of $g t f R$ expression only on titanium surfaces, suggesting that similar to $g t f B$ and $g t f C, g t f R$ can be more actively transcribed when cells are adhering to this type of solid surface in the presence of sucrose [42]. The late $(24 \mathrm{~h})$ timing of this upregulation on titanium is consistent with the timing of $g t f B$ and $g t f C$ upregulation on hydroxyapatite surfaces [24]. Titanium surfaces were also associated with increased $C$. albicans biomass in mixed compared with single biofilms. It is possible that the physical and chemical properties of this biomaterial can affect protein adsorption on the surface modulating secondary fungal adhesion and biofilm formation [43-45]. Enhanced growth of C. albicans on titanium surfaces induced by pioneer dental plaque species such as $S$. oralis may promote mucosal inflammation and peri-implant disease and increased dietary sucrose exposure can amplify these effects [46].

Among the Gtf enzymes synthesized by $S$. mutans, only GtfB binds avidly to $C$. albicans and mediates coaggregation interactions [32]. The selective binding of $\mathrm{GtfB}$ to $C$. albicans was suggested to be mediated by the carboxyl terminus of this protein, which contains the GBD and differs among the three Gtfs [32, 47]. Glucan-binding Gtf protein domains bind to $\alpha$-1,6-linked glucosyl residues in glucans which confer a specific structural motif recognized as a binding site $[48,49]$. GtfB binding to $C$. albicans is primarily mediated by mannans or mannoproteins exposed on the fungal cell wall [41]; however, the role of the GBD and how it affects selectivity of this Gtf in binding has never been examined. In our studies rGBD was not significantly adsorbed on the surface of $C$. albicans. This could be due to reduced Candida-binding function of the GtfR GBD or the requirement of a conformational structure for binding that is not attainable by producing the protein fragment in an E. coli host. Because there was also no detectable adsorption of the purified GtfR to Candida, direct binding may not play a major role in these interactions.

In conclusion, for the first time we showed that GtfR produces $\alpha$-glucans which mediate cross-kingdom biofilm interactions between $C$. albicans and $S$. oralis. GtfR increased biofilm formation by a positive effect on bacterial biofilm matrix and biomass. This effect was modulated by the type of substratum and the mucosal environment in vivo.

Acknowledgements This study was supported by NIH grants R01 DE013986 to ADB, R01AI029735, R21AI128379, R21AI126146, R21AIAI139940 to MJC, and the Brazilian funding agency FAPESP (2017/21289-0).

\section{Compliance with ethical standards}

Conflict of interest The authors declare that they have no conflict of interest.

Publisher's note Springer Nature remains neutral with regard to jurisdictional claims in published maps and institutional affiliations.

Open Access This article is licensed under a Creative Commons Attribution 4.0 International License, which permits use, sharing, adaptation, distribution and reproduction in any medium or format, as long as you give appropriate credit to the original author(s) and the source, provide a link to the Creative Commons license, and indicate if changes were made. The images or other third party material in this article are included in the article's Creative Commons license, unless indicated otherwise in a credit line to the material. If material is not included in the article's Creative Commons license and your intended use is not permitted by statutory regulation or exceeds the permitted use, you will need to obtain permission directly from the copyright holder. To view a copy of this license, visit http://creativecommons. org/licenses/by/4.0/. 


\section{References}

1. Vacca-Smith AM, Venkitaraman AR, Quivey RG Jr, Bowen WH. Interactions of streptococcal glucosyltransferases with alphaamylase and starch on the surface of saliva-coated hydroxyapatite. Arch Oral Biol. 1996;41:291-8.

2. Bowen WH, Koo H. Biology of Streptococcus mutans-derived glucosyltransferases: role in extracellular matrix formation of cariogenic biofilms. Caries Res. 2011;45:69-86.

3. Kopec LK, Vacca-Smith AM, Bowen WH. Structural aspects of glucans formed in solution and on the surface of hydroxyapatite. Glycobiology. 1997;7:929-34.

4. Costerton JW, Cheng KJ, Geesey GG, Ladd TI, Nickel JC, Dasgupta M, et al. Bacterial biofilms in nature and disease. Annu Rev Microbiol. 1987;41:435-64.

5. Schilling KM, Bowen WH. Glucans synthesized in situ in experimental salivary pellicle function as specific binding sites for Streptococcus mutans. Infect Immun. 1992;60:284-95.

6. Paes Leme AF, Koo H, Bellato CM, Bedi G, Cury JA. The role of sucrose in cariogenic dental biofilm formation-new insight. J Dent Res. 2006;85:878-87.

7. Flemming HC, Wingender J. The biofilm matrix. Nat Rev Microbiol. 2010;8:623-33.

8. Bowen WH, Burne RA, Wu H, Koo H. Oral biofilms: pathogens, matrix, and polymicrobial interactions in microenvironments. Trends Microbiol. 2018;26:229-42.

9. Rickard AH, Gilbert P, High NJ, Kolenbrander PE, Handley PS. Bacterial coaggregation: an integral process in the development of multi-species biofilms. Trends Microbiol. 2003;11:94-100.

10. Diaz PI, Chalmers NI, Rickard AH, Kong C, Milburn CL, Palmer $\mathrm{RJ} \mathrm{Jr}$, et al. Molecular characterization of subject-specific oral microflora during initial colonization of enamel. Appl Environ Microbiol. 2006;72:2837-48.

11. Diaz PI, Dupuy AK, Abusleme L, Reese B, Obergfell C, Choquette $\mathrm{L}$, et al. Using high throughput sequencing to explore the biodiversity in oral bacterial communities. Mol Oral Microbiol. 2012;27:182-201.

12. Vickerman MM, Clewell DB, Jones GW. Sucrose-promoted accumulation of growing glucosyltransferase variants of Streptococcus gordonii on hydroxyapatite surfaces. Infect Immun. 1991;59:3523-30.

13. Fujiwara T, Hoshino T, Ooshima T, Sobue S, Hamada S. Purification, characterization, and molecular analysis of the gene encoding glucosyltransferase from Streptococcus oralis. Infect Immun. 2000;68:2475-83.

14. Sulavik MC, Clewell DB. Rgg is a positive transcriptional regulator of the Streptococcus gordonii gtfG gene. J Bacteriol. 1996;178:5826-30.

15. Whitmore SE, Lamont RJ. The pathogenic persona of communityassociated oral streptococci. Mol Microbiol. 2011;81:305-314.19.

16. Ricker A, Vickerman M, Dongari-Bagtzoglou A. Streptococcus gordonii glucosyltransferase promotes biofilm interactions with Candida albicans. J Oral Microbiol. 2014;6:23419. https://doi.org/ 10.3402/jom.v6.23419.

17. $\mathrm{Xu} \mathrm{H}$, Jenkinson HF, Dongari-Bagtzoglou A. Innocent until proven guilty: mechanisms and roles of Streptococcus-Candida interactions in oral health and disease. Mol Oral Microbiol. 2014;29:99-116.

18. Xu H, Sobue T, Thompson A, Xie Z, Poon K, Ricker A, et al. Streptococcal co-infection augments Candida pathogenicity by amplifying the mucosal inflammatory response. Cell Microbiol. 2014;16:214-31.

19. Bertolini MM, Xu H, Sobue T, Nobile CJ, Del Bel Cury AA, Dongari-Bagtzoglou A. Candida-streptococcal mucosal biofilms display distinct structural and virulence characteristics depending on growth conditions and hyphal morphotypes. Mol Oral Microbiol. 2015;30:307-22.

20. Frade JP, Arthington-Skaggs BA. Effect of serum and surface characteristics on Candida albicans biofilm formation. Mycoses. 2011;54:e154-162.

21. Vickerman MM, Sulavik MC, Minick PE, Clewell DB. Changes in the carboxyl-terminal repeat region affect extracellular activity and glucan products of Streptococcus gordonii glucosyltransferase. Infect Immun. 1996;64:5117-28.

22. Xu H, Sobue T, Bertolini M, Thompson A, Dongari-Bagtzoglou A. Streptococcus oralis and Candida albicans synergistically activate $\mu$-calpain to degrade E-cadherin from oral epithelial junctions. J Infect Dis. 2016;214:925-34.

23. Xu H, Sobue T, Bertolini M, Thompson A, Vickerman M, Nobile $\mathrm{CJ}$, et al. S. oralis activates the Efg1 filamentation pathway in $C$. albicans to promote cross-kingdom interactions and mucosal biofilms. Virulence. 2017;8:1602-17.

24. Falsetta ML, Klein MI, Colonne PM, Scott-Anne K, Gregoire S, Pai $\mathrm{CH}$, et al. Symbiotic relationship between Streptococcus mutans and Candida albicans synergizes virulence of plaque biofilms in vivo. Infect Immun. 2014;82:1968-81.

25. Dongari-Bagtzoglou A, Kashleva H. Development of a highly reproducible three-dimensional organotypic model of the oral mucosa. Nat Protoc. 2006;1:2012-8.

26. Dongari-Bagtzoglou A, Kashleva H. Development of a novel three-dimensional in vitro model of oral Candida infection. Micro Pathog. 2006;40:271-8.

27. Klein MI, Duarte S, Xiao J, Mitra S, Foster TH, Koo H. Structural and molecular basis of the role of starch and sucrose in Streptococcus mutans biofilm development. Appl Environ Microbiol. 2009;75:837-41.

28. Amann RI, Binder BJ, Olson RJ, Chisholm SW, Devereux R, Stahl DA. Combination of 16S ribosomal RNA-targeted oligonucleotide probes with flow cytometry for analyzing mixed microbial populations. Appl Env Microbiol. 1990;56:1919-25.

29. Dongari-Bagtzoglou A, Kashleva H, Dwivedi P, Diaz P, Vasilakos J. Characterization of mucosal Candida albicans biofilms. PLoS ONE. 2009;4:e7967.

30. Cury JA, Koo H. Extraction and purification of total RNA from Streptococcus mutans biofilms. Anal Biochem. 2007;365:208-14.

31. He J, Kim D, Zhou X, Ahn SJ, Burne RA, Richards VP, et al. RNA-Seq reveals enhanced sugar metabolism in Streptococcus mutans co-cultured with Candida albicans within mixed-species biofilms. Front Microbiol. 2017;8:1036.

32. Gregoire S, Xiao J, Silva BB, Gonzalez I, Agidi PS, Klein MI, et al. Role of glucosyltransferase B in interactions of Candida albicans with Streptococcus mutans and with an experimental pellicle on hydroxyapatite surfaces. Appl Environ Microbiol. 2011;77:6357-67.

33. Bertolini M, Ranjan A, Thompson A, Diaz PI, Sobue T, Maas K, et al. Candida albicans induces mucosal bacterial dysbiosis that promotes invasive infection. PLoS Pathog. 2019;15:e1007717.

34. Cavalcanti YW, Wilson M, Lewis M, Del-Bel-Cury AA, da Silva WJ, Williams DW. Modulation of Candida albicans virulence by bacterial biofilms on titanium surfaces. Biofouling. 2016; 32:123-34

35. Kim D, Liu Y, Benhamou RI, Sanchez H, Simón-Soro Á, Li Y, et al. Bacterial-derived exopolysaccharides enhance antifungal drug tolerance in a cross-kingdom oral biofilm. ISME J. 2018;12:1427-42.

36. Maidan MM, Thevelein JM, Van Dijck P. Carbon source induced yeast-to-hypha transition in Candida albicans is dependent on the presence of amino acids and on the G-protein-coupled receptor Gpr1. Biochem Soc Trans. 2005;33:291-3.

37. Uppuluri P, Chaturvedi AK, Srinivasan A, Banerjee M, Ramasubramaniam AK, Kohler JR, et al. Dispersion as an important 
step in the Candida albicans biofilm developmental cycle. PLoS Pathog. 2010;6:e1000828.

38. Laffin M, Fedorak R, Zalasky A, Park H, Gill A, Agrawal A, et al. A high-sugar diet rapidly enhances susceptibility to colitis via depletion of luminal short-chain fatty acids in mice. Sci Rep. 2019;9:12294.

39. Poupet C, Saraoui T, Veisseire P, Bonnet M, Dausset C, Gachinat $\mathrm{M}$, et al. Lactobacillus rhamnosus Lcr35 as an effective treatment for preventing Candida albicans infection in the invertebrate model Caenorhabditis elegans: first mechanistic insights. PLoS ONE. 2019;6:e216184. 14

40. Koo H, Andes DR, Krysan DJ. Candida-streptococcal interactions in biofilm-associated oral diseases. PLoS Pathog. 2018;14: e1007342.

41. Hwang G, Liu Y, Kim D, Li Y, Krysan DJ, Koo H. Candida albicans mannans mediate Streptococcus mutans exoenzyme GtfB binding to modulate cross-kingdom biofilm development in vivo. PLoS Pathog. 2017;13:e1006407.

42. Hudson MC, Curtiss R 3rd. Regulation of expression of Streptococcus mutans genes important to virulence. Infect Immun. 1990;58:464-70.

43. Fürst MM, Salvi GE, Lang NP, Persson GR. Bacterial colonization immediately after installation on oral titanium implants. Clin Oral Implants Res. 2007;18:501-8.
44. Abdallah MN, Tran SD, Abughanam G, Laurenti M, Zuanazzi D, Mezour MA, et al. Biomaterial surface proteomic signature determines interaction with epithelial cells. Acta Biomater. 2017;54:150-63.

45. Pantaroto HN, Amorim KP, Matozinho Cordeiro J, Souza JGS, Ricomini-Filho AP, Rangel EC, et al. Proteome analysis of the salivary pellicle formed on titanium alloys containing niobium and zirconium. Biofouling. 2019. https://doi.org/10.1080/08927014. 2019.1580360.

46. Souza JGS, Cury JA, Ricomini Filho AP, Feres M, Faveri M, Barão VAR. Effect of sucrose on biofilm formed in situ on titanium material. J Periodontol. 2019;90:141-8.

47. Koo H, Xiao J, Klein MI, Jeon JG. Exopolysaccharides produced by Streptococcus mutans glucosyltransferases modulate the establishment of microcolonies within multispecies biofilms. J Bacteriol. 2010;192:3024-32.

48. Buchan RA, Jenkinson HF. Glucosyltransferase production by Streptococcus sanguis Challis and comparison with other oral streptococci. Oral Microbiol Immunol. 1990;5:63-71.

49. Kobayashi S, Koga K, Hayashida O, Nakano Y, Hasegawa Y. Glucan-binding domain of a glucosyltransferase from Streptococcus sobrinus: isolation of a 55-kilodalton peptide from a trypsin digest of glucosyltransferase prebound to insoluble glucan. Infect Immun. 1989;57:2210-3. 